UNIVERSIDADE ESTADUAL PAULISTA "JÚLIO DE MESQUITA FILHO" FACULDADE DE CIÊNCIAS/CAMPUS DE BAURU

PROGRAMA DE PÓS-GRADUAÇÃO EM CIÊNCIAS DE TECNOLOGIAS DE MATERIAIS

\title{
DINÂMICA DE VÓRTICES EM FILMES FINOS SUPERCONDUTORES DE SUPERFÍCIE VARIÁVEL
}

\author{
Mauro Cesar Videira Pascolati
}

Orientadores:

Edson Sardella

e

Paulo Noronha Lisboa Filho

BAURU

2010 
Mauro Cesar Videira Pascolati

DINÂMICA DE VÓRTICES

EM FILMES FINOS SUPERCONDUTORES

DE SUPERFÍCIE VARIÁVEL

Dissertação apresentada ao programa de Pós-Graduação em Ciência e Tecnologia de Materiais da Universidade Estadual Paulista como parte dos pré-requisitos para a obtenção do título de Mestre. 


\section{UNIVERSIDADE ESTADUAL PAULISTA \\ "JÜLIO DE MESQUITA FILHO" \\ CAMPUS DE BAURU \\ FACULDADE DE CIENCIAS DE BAURU}

ATA DA DEFESA PÚBLICA DA DISSERTAÇĀO DE MESTRADO DE MAURO CESAR VIDEIRA PASCOLATI, DISCENTE DO PROGRAMA DE PÓS-GRADUAÇĀO EM CIÊNCIA E TECNOLOGIA DE MATERIAIS, DO(A) FACULDADE DE CIENCIAS DE BAURU.

Aos 28 dias do mês de abril do ano de 2010, às 14:00 horas, no(a) Anfiteatro da Pós-graduação/FC, reuniu-se a Comissão Examinadora da Defesa Pública, composta pelos seguintes membros: Prof. Dr. EDSON SARDELLA do(a) Departamento de Fisica / Faculdade de Ciencias de Bauru, Prof. Dr. WILSON AIRES ORTIZ do(a) Departamento de Fisica / Universidade Federal de São Carlos, Prof. Dr. CLECIO CLEMENTE DE SOUZA SILVA do(a) Departamento de Fisica / Universidade Federal de Pernambuco, sob a presidência do primeirro, a fim de proceder a argüição pública da DISSERTAÇĀO DE MESTRADO de MAURO CESAR VIDEIRA PASCOLATI, intitulado "DINÂMICA DE VÓRTICES EM FILMES FINOS SUPERCONDUTORES DE SUPERFICIE VARIÁVEL". Após a exposiçã̃o, o discente foi argüido oralmente pelos membros da Comissão Examinadora, tendo recebido o conceito

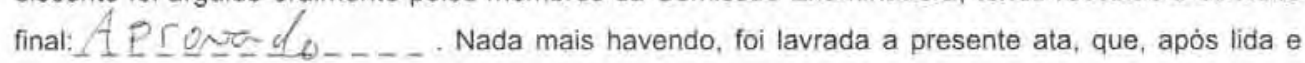
aprovada, foi assinada pelos membros da Comissão Examinadora.

Prof. Dr. EDSON SARDELLA

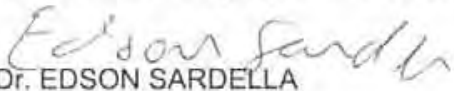

$$
\text { lutsitis }
$$

Prof, Dr. WILSON AIRES ORTIZ

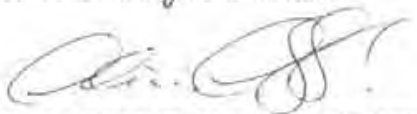

Prof. Dr. CLECIO CLEMENTE DE SOUZA SILVA 


\section{Dedicatória}

À minha esposa, Mariana Martins Ricardo, por acreditar na minha capacidade muito mais do que eu mesmo acredito. Por ver em mim qualidades que não vejo e o mais importante, por estar ao meu lado em todos os momentos.

À minha mãe, Eunice Videira Pascolati, por me acolher cegamente em sua vida, desde o momento que me tomou nos braços pela primeira vez.

A meu pai, Osni Pascolati, por me estender suas mãos, mesmo em momentos de grandes dificuldades, quando na minha imaturidade ou inocência mais precisei. 


\section{Agradecimentos}

A meu orientador, Edson Sardella, pela sinseridade, boa vontade e muita paciência que teve comigo no desenvolvimento deste trabalho.

A meu co-orientador, Paulo Noronha Lisboa Filho, por me proporcionar esta oportunidade de trabalho, que certamente provocará em minha vida mudanças substanciais.

E finalmente a Deus, por colocar em meu caminho, todas essas pessoas maravilhosas, que sempre me apoiaram, pois sem Ele, nada disso seria possível. 


\section{Resumo}

O interesse em conhecer o comportamento supercondutor tem sido cada vez maior nas últimas décadas. Na busca de melhores características supercondutoras, descobriuse que amostras volumétricas apresentam características muito diferentes de amostras mesoscópicas (amostras com dimensões próximas dos comprimentos de penetração de London e coerência). Como exemplo, podemos citar a não formação da rede de Abrikosov, como consequência do efeito de confinamento (efeito associado às dimensões reduzidas da amostra) e também uma mudança considerável nos valores dos campos críticos.

Neste trabalho foram resolvidas as equações de Ginzburg-Landau dependentes do tempo (TDGL), para fazer uma análise detalhada da dinâmica de vórtices em filmes finos mesoscópicos. Para resolvê-las, utilizamos o método das variáveis de ligação com invariância de calibre, adaptado para o algoritmo de diferenças finitas, utilizado para obter a densidade dos pares de Cooper e também curvas de magnetização.

O estudo dessa dinâmica de vórtices, foi feito em três amostras com superfícies geométricas diferentes (côncava, convexa e rugosa). Observamos que na comparação entre as duas primeiras, há uma diferança considerável nos valores dos campos críticos, bem como no comportamento da magnetização comparado com um filme plano. 
Já para a amostra de superfície rugosa, observamos que existe uma competição entre o efeito de confinamento e a rugosidade em relação à configuração dos vórtices.

Apresentamos também, uma tabela que mostra resumidamente os estados estacionários dos vórtices nas três amostras.

Palavras chaves: Supercondutividade, vórtices, equações de Ginzburg-Landau, método das variáveis de ligação.

Esta dissertação se apresenta da seguinte forma:

- No Capítulo 1, fazemos um breve relato histórico do fenômeno da supercondutividade, desde sua descoberta feita por Onnes em 1911, até os supercondutores cerâmicos de alta temperatura crítica descobertos há mais de duas décadas, e apresentamos algumas aplicações.

- No Capítulo 2, Apresentamos o desenvolvimento das teorias fenomenológicas de London e Ginzburg-Landau, evidenciando as propriedades supercondutoras que cada modelo é capaz de contemplar. Esta revisão teórica é feita considerando um supercondutor na presença de um campo magnético externo aplicado, apresentando o estado de vórtices e as características de um vórtice isolado.

- No Capítulo 3, apresentamos o desenvolvimento do método de discretização das equações de Ginzburg-Landau dependentes do tempo, levando em conta neste processo a invariância de calibre e discutindo as condições de contorno necessárias para a solução das equações. Finalmente fizemos a adaptação das equações de Ginzburg-Landau dependentes do tempo para filmes finos.

- No Capítulo 4, apresentamos a descrição das topologias (amostras) estudadas, introduzindo os parâmetros geométricos e discutindo os detalhes das simulações numéricas. Em seguida, fazemos a apresentação dos resultados e dis- 
cussões, com relação aos comportamentos da magnetização, dos campos críticos e da dinâmica dos vórtices.

- No Capítulo 5, finalmente apresentamos as conclusões gerais e perspectivas da presente pesquisa. 


\begin{abstract}
The interest to investigate the behavior of a superconductor has grown in the last few decades. Having in mind to search for better superconducting characteristics, it has been found that bulk samples present characteristics much more different than mesoscopic samples (samples with dimensions of the same order of the London penetration length and the coherence length). As an example, we can mention the non-formation of an Abrikosov vortex lattice as a consequence of the confinement effect (effect associated with the reduced dimensions of the sample) and also considerable change in the critical field values.

In the present work we solved the time dependent Ginzburg-Landau equations (TDGL), in order to make a detailed analysis of the vortex dynamics in mesoscopic thin films. To solve these equations, we have used the link variables method which is gauge invariant. From this, we obtain the Cooper pair density and the magnetization curves.

The vortex dynamics was investigated for three different surfaces of the film (concave, convex, and irregular). We have observed that, with respect to the parabolic geometries, there is a considerable difference for the critical fields, as well as for the behavior of the magnetization compared to a flat film. On the other hand, for a sample with an irregular surface, we have seen that there is a competition between the confinement effect and rugosity with respect to vortex configurations.
\end{abstract}


We also present a table which summarizes the vortex stationary states for the three topologies mentioned above.

Key words: Superconductivity, vortices, Ginzburg-Landau equations, link variables method.

This dissertation is presented in the following way:

- In Chapter 1, we make a brief historical survey of the superconducting phenomenon, since its discovery made by Onnes in 1911 until the ceramic superconductors of high critical temperature discovered more than two decades ago, and present some applications.

- In Chapter 2, we present the development of the phenomenological theories of London and Ginzburg-Landau showing which superconducting properties each one can describe. The theoretical review is made considering a superconductor in the presence of an external applied magnetic field. The vortex state and the characteristics of an isolated vortex are discussed.

- In Chapter 3, we present the development of the link variables method in order to discretize the time dependent Ginzburg-Landau equations (TDGL). The gauge invariance and the boundary conditions are taken into account. Finally, we made an adaptation of the time dependent Ginzburg-Landau equations for thin films.

- In Chapter 4, we present the description of the studied topologies (samples) by introducing the geometrical parameters and discussing the details of the numerical simulations. Next, we make a presentation of the results and discussion with respect to the behavior of the magnetization, the critical fields, and the vortex dynamics. 
- In Chapter 5, finally we present the conclusions and perspectives of this research. 


\section{Sumário}

1 Introdução $\quad 11$

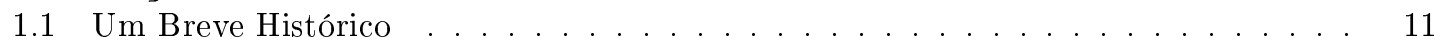

1.2 O Fenômeno da Supercondutividade . . . . . . . . . . . . . . . . 12

2 Teorias Fenomenológicas da Supercondutividade 19

2.1 Introdução . . . . . . . . . . . . . . . . . . . . . . . . . . . . . . . . . . . . . . . . . . . . . . . . . .

2.2 O Modelo de London . . . . . . . . . . . . . . . . . . . . . . . . . . . . . . . . 19

2.3 A Teoria de Ginzburg-Landau . . . . . . . . . . . . . . . . . . . . . . . . . . . . . . . . . . . . . . . . . . . . .

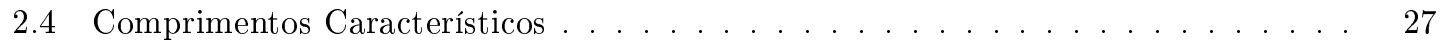

2.4.1 Comprimento de Coerência . . . . . . . . . . . . . . . 27

2.4.2 Comprimento de Penetração de London . . . . . . . . . . . . . . . . . 29

2.5 Campos Críticos . . . . . . . . . . . . . . . . . . . . . . . . . . . . . . . . . . . . 30

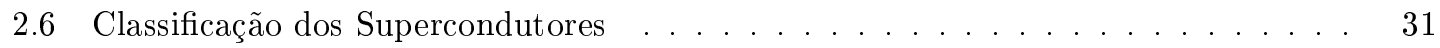

2.7 Diagrama de Fase . . . . . . . . . . . . . . . . . . . . . . . . . . . . . . . . . . . . . . . . . . . . . . . . . . . .

2.8 Quantização de fluxo . . . . . . . . . . . . . . . . . . . . . . . . . . . . . . . . . . . . . . . . .

2.9 Estrutura de um Vórtice . . . . . . . . . . . . . . . . . . . . . . . . . . . . . . . . . . . . . . . . . 37

2.10 Vórtices em Supercondutores Macroscópicos . . . . . . . . . . . . . . . . . . . . . . . . . . . . 38

2.11 Supercondutividade Mesoscópica . . . . . . . . . . . . . . . . 39

3 Método de Variáveis de Ligação para as Equações TDGL 42

3.1 Introdução . . . . . . . . . . . . . . . . . . . . . . . . . . . . . . . . . . . . . . . . . . . . . . . . . . . . .

3.2 As Equações TDGL . . . . . . . . . . . . . . . . . . . . . . . . . . . . . . . 43

3.2 .1 Método das Variáveis de Ligação . . . . . . . . . . . . . . . . . . . . . . . . 45

3.2 .2 Condições de Contorno . . . . . . . . . . . . . . . . . . . . . . . . . . . . 52

3.3 Filmes de Superfície Variável . . . . . . . . . . . . . . . . . . . 54

4 Estudo de Algumas Topologias de Filmes Finos Supercondutores 5

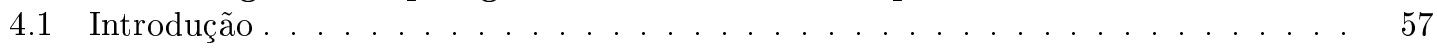

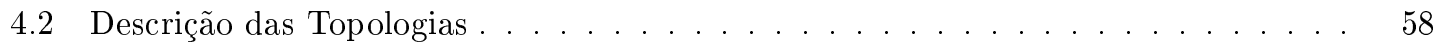

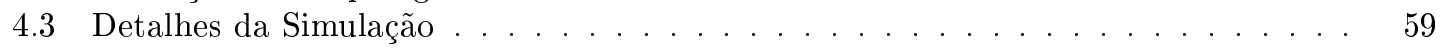

4.4 Resultados e Discussões . . . . . . . . . . . . . . . . . . . . 62

4.4.1 Comportamento dos Campos Críticos Inferior e Superior . . . . . . . . . . 62

4.4 .2 Comportamento da Magnetização . . . . . . . . . . . . . . . . 64

4.4.3 Dinâmica de Vórtices . . . . . . . . . . . . . . . 66

5 Conclusões $\quad 75$ 


\section{Capítulo 1}

\section{Introdução}

\subsection{Um Breve Histórico}

O desenvolvimento científico e tecnológico, desde o início da revolução industrial, deu um salto muito grande e o modo de vida social sofreu profundas alterações. Entusiasmados com a avalanche de novas descobertas, os cientistas, por assim dizer, investiram massivamente em busca de novas descobertas, sobretudo na área de novos materiais, que são de vital importância para uso industrial. Dentre os muitos fenômenos que foram descobertos no final do século XIX e início do século XX, certamente a supercondutividade ganhou um destaque especial da comunidade científica. A possibilidade de termos um metal conduzindo energia elétrica sem apresentar efeito Joule, era simplesmente fascinante. Entretanto, conforme usualmente ocorre, havia enormes obstáculos entre a descoberta do fenômeno e aplicação para uso científico e tecnológico. No caso da supercondutividade a dificuldade era a temperatura, pois Onnes em 1911 descobriu este fenômeno ao resfriar o mercúrio a aproximadamente 4.2 K. [1] Temperaturas tão baixas como esta tornavam inviável as aplicações deste material. Desde então, grandes esforços foram envidados para obter um modelo que explicasse de forma satisfatória tal fenômeno e também descobrir diferentes materiais que manifestassem o fenômeno da supercondutividade a temperaturas mais elevadas. 
Em 1935 surge um modelo fenomenológico (descritivo) proposto pelos irmãos London. [2] Alguns anos mais tarde, em 1950, Ginzburg e Landau [3] propõem um modelo também puramente fenomenológico. Ambas as teorias eram capazes de descrever o comportamento de um supercondutor em nível macroscópico. Em 1957 surge uma teoria microscópica, batizada como teoria BCS (devido aos seus autores Bardeen, Cooper e Scheriffer), onde se introduziu o conceito de pares de Cooper. $[4,5]$ Esta teoria conseguia explicar satisfatoriamente os mecanismos mecânicoquânticos responsáveis pela supercondutividade.

Em 1985 surgem os supercondutores cerâmicos de altas temperaturas críticas, materiais considerados isolantes. As teorias fenomenológicas de Ginzburg-Landau e London ainda podem ser empregadas para a descrição dos materiais cerâmicos. [6] Porém, ainda não surgiu uma teoria microscópica que explique o fenômeno quanticamente.

Apesar de a supercondutividade ser um fenômeno de baixas temperaturas a sua utilização hoje em dia já é uma realidade e citaremos alguns exemplos na próxima Seção.

\subsection{O Fenômeno da Supercondutividade}

Em 1908 H. Kammerlingh Onnes, [1] em Leiden na Holanda, deu um passo importante no estudo da física de baixas temperaturas, liquefazendo o Hélio em seu laboratório. Três anos depois, Onnes observou que ao resfriar uma amostra de mercúrio sólido, sua resistência caía abruptamente e aparentemente ${ }^{1}$ por completo a 4.2 K (ver Figura 1.1). A essa temperatura deu-se o nome mais tarde de temperatura de transição, ou temperatura crítica $T_{c}$, que era aquela em que se observava a transição do estado normal ao novo estado, que o próprio Onnes mais tarde chamou de

\footnotetext{
${ }^{1}$ Atualmente é sabido que, num supercondutor podem circular correntes que persistem durante anos sem que se possa detectar seus decaimentos.
} 
supercondutor.

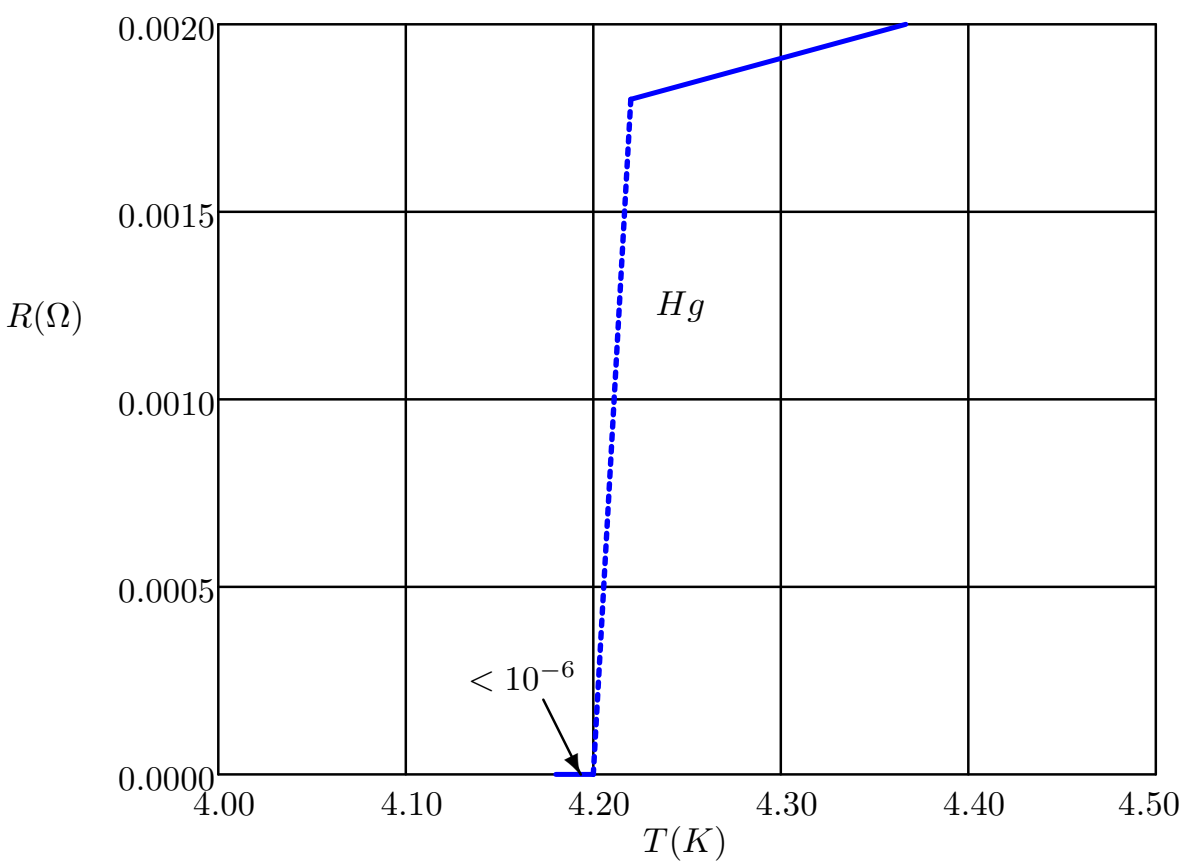

Figura 1.1: Gráfico histórico de Onnes: resistência do mercúrio sólido em função da temperatura.

Entretanto, percebeu-se mais adiante que, além da resistividade nula, a supercondutividade tem outra importante assinatura. A supercondutividade também se limita a um certo valor de densidade de corrente crítica $J_{c}$ e um campo magnético crítico $H_{c}$ aplicado, sendo que estes valores são característicos de cada material. A Figura 1.2 ilustra a transição entre o estado normal e o supercondutor e as correspondentes grandezas críticas.

Outros aspectos fascinantes da supercondutividade viriam a ser descobertos posteriormente à descoberta de Onnes. Em 1933 Walther Meissner e Robert Ochsenfeld [7] descobriram que um supercondutor, quando encontra-se abaixo de $T_{c}$, submetido a um campo magnético abaixo de $H_{c}$, expulsa totalmente o campo magnético de seu interior, tendo portanto um comportamento diamagnético perfeito. A este novo fenômeno deu-se o nome de efeito Meissner. Experimentos demonstraram que um 


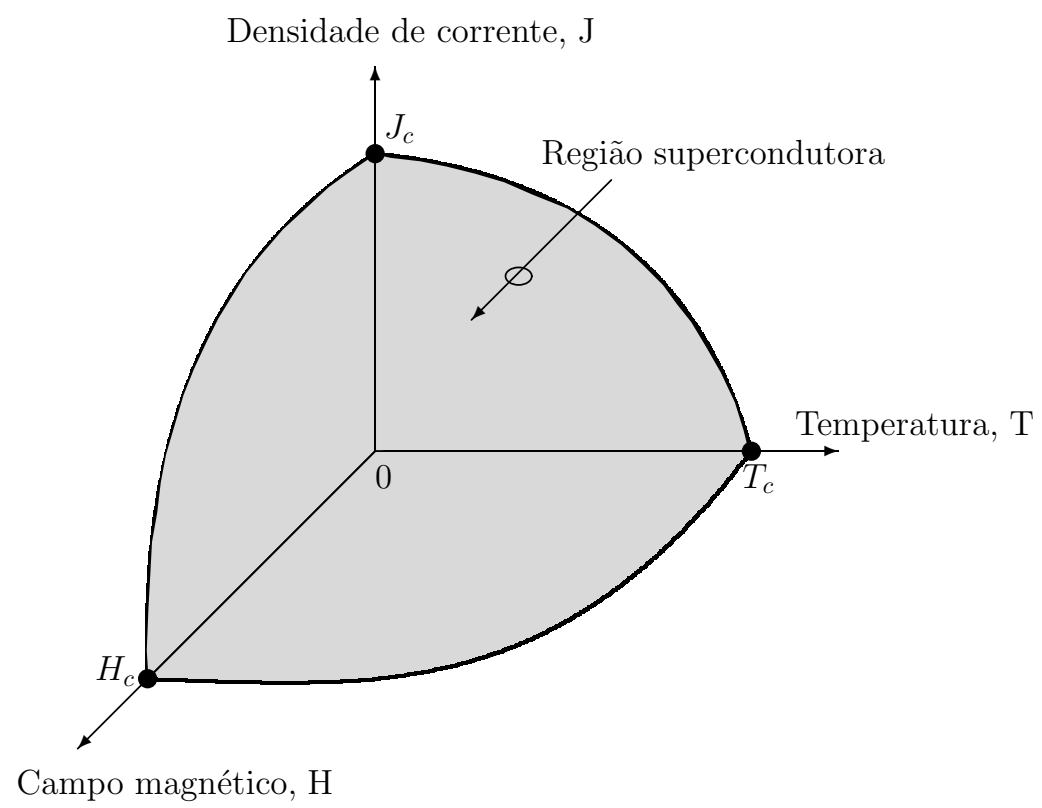

Figura 1.2: Diagrama de fase dos primeiros supercondutores descobertos por Onnes.

supercondutor era mais do que um condutor perfeito, pois este último, dependia da história magnética do material. Um supercondutor imerso em um campo magnético abaixo de $H_{c}$ quando resfriado até uma determinada temperatura abaixo de $T_{c}$ expulsa totalmente o campo magnético. O mesmo não ocorre com um condutor perfeito. A Figura 1.3 ilustra este cenário.

Em 1935 os irmãos F. e H. London [2] propuseram uma teoria fenomenológica, que consistia num modelo simples de dois fluidos (o modelo de London). Esta teoria descrevia o efeito Meissner e também o comprimento de penetração $\lambda$, que pode ser compreendido como sendo uma penetração superficial de campo magnético no supercondutor. Eles concluíram que esta penetração tinha um comportamento exponencial, decaindo da superfície para o interior do supercondutor. Muito embora não conhecendo os mecanismos microscópicos da supercondutividade, esta teoria descrevia satisfatoriamente o comportamento do campo magnético e das correntes dentro de um supercondutor.

Em 1950 Vitaly Gingburg e Lev Landau [3] propuseram uma nova teoria fenome- 


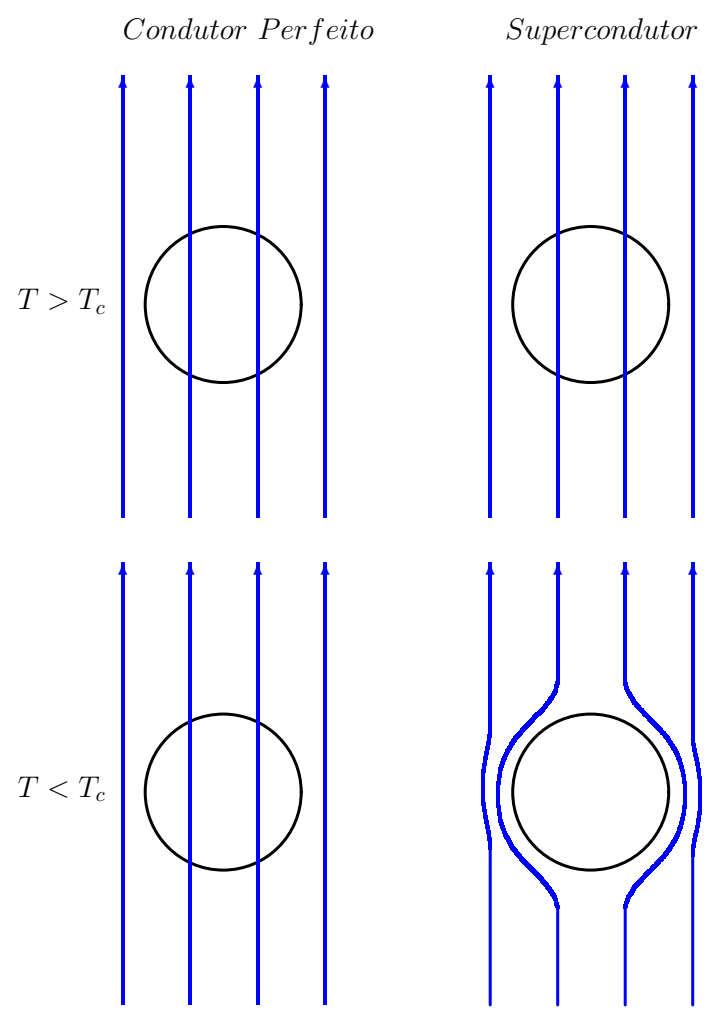

Figura 1.3: Condutor perfeito e supercondutor.

nológica que descrevia detalhadamente em nível macroscópico o comportamento de um supercondutor próximo à temperatura crítica. Utilizando um parâmetro de ordem complexo $\psi(\mathbf{r})$ e o potencial vetor $\mathbf{A}(\mathbf{r})$, eles propuseram uma forma para a energia do supercondutor a qual era representativa deste estado. Nesta teoria, o potencial vetor está relacionado com o campo magnético local por meio da relação $\mathbf{B}=\boldsymbol{\nabla} \times \mathbf{A}$, e $|\psi|^{2}$ representa a densidade do estado supercondutor. Ginzburg e Landau foram capazes de prever o comprimento de penetração de London $\lambda$ e introduziram um novo comprimento fundamental da supercondutividade chamado de comprimento de coerência $\xi$, relacionado à variação espacial de $\psi(\mathbf{r})$. Embora estes comprimentos fundamentais da teoria dependam de $T$, a razão $\kappa=\lambda(T) / \xi(T)$ não depende. Essa teoria também previa a existência de dois tipos de supercondutores, a partir do parâmetro de Ginzburg-Landau $\kappa$ : tipo-I $(\kappa<1 / \sqrt{2})$ e tipo-II 
$(\kappa>1 / \sqrt{2})$.

Em 1957, Alexei Abrikosov [8] estudou os supercondutores quando submetidos a campos magnéticos, descrevendo o comportamento dos supercondutores do tipo-I e do tipo-II. Ele propôs que os supercondutores do tipo-I expulsam totalmente o fluxo magnético até que o campo aplicado alcance um determinado valor chamado campo crítico $H_{c}(T)$. Uma vez que o campo aplicado excede este valor crítico, o supercondutor retorna ao seu estado normal. O campo crítico depende da temperatura: quanto mais baixa a temperatura, maior o seu valor; é máximo em $T=0$ e nulo em $T=T_{c}$. Abrikosov foi além, e propôs que existia uma outra classe de materiais, os do tipo-II, os quais se comportam de forma ligeiramente diferente. Nestes materiais, existe um segundo campo crítico $H_{c 2}(T)$ o qual é maior que o primeiro campo crítico $H_{c 1}(T)$. Abaixo do campo crítico inferior $H_{c 1}(T)$ o supercondutor encontra-se no estado Meissner. Quando o campo aplicado ultrapassa este valor, o supercondutor não expulsa mais o fluxo magnético por completo. No entanto, o supercondutor não retorna ao estado normal. Quando o campo aplicado encontra-se entre os campos críticos inferior e superior, ocorre a penetração parcial de fluxo magnético, onde a amostra desenvolve uma estrutura, consistindo de regiões normais e supercondutoras. Este estado é conhecido como estado misto. Nesse estado, a presença do fluxo magnético no interior do supercondutor se dá na forma de vórtices, objetos formados de regiões normais em seu interior e supercondutoras ao seu redor. Esses vórtices são pacotes de fluxo magnético quantizados atravessando a amostra, os quais, num supercondutor homogêneo, se distribuem de forma regular. Alexei Abrikosov propôs que esta distribuição regular dos vórtices era de forma quadrada. Posteriormente demonstrou-se que a rede triangular era a mais estável. Este último cenário foi comprovado por experimentos de decoração magnética.[9]

Somente em 1957 apareceu uma teoria microscópica capaz de explicar de forma 
satisfatória o fenômeno da supercondutividade a baixas temperaturas. Esta teoria foi elaborada por John Bardeen, Leon Cooper e Robert Schrieffer e em homenagem a eles, esta teoria recebeu o nome de BCS. [4] Esta teoria é baseada na interação de uma nuvem eletrônica com as vibrações elásticas da rede cristalina. Mesmo sabendo que entre os elétrons ocorre uma interação Coulombiana repulsiva, a baixas temperaturas é possível que de uma forma indireta ocorra atração entre dois elétrons via vibração da rede (os fônons). Dois elétrons conectados desta maneira indireta formam os chamados pares de Cooper. [5] Para um supercondutor de baixas temperaturas, quando estes pares estão sob a influência de um campo elétrico, entram em movimento, só que de forma altamente ordenada, ou seja, todos os pares se movem da mesma forma. Desta maneira altamente ordenada, os pares se movimentam pela rede sem sofrer espalhamentos aleatórios produzidos pelas imperfeições da rede que provocam a resistência elétrica a baixas temperaturas.

Alguns anos mais tarde, L. P. Gorkov [10] demonstrou que a teria de GinzburgLandau emerge naturalmente da teoria BCS. A partir de então, teve-se a certeza de que $|\psi|^{2}$ representa a densidade de pares de Cooper.

Do ponto de vista quântico, é sabido que os elétrons tendo spin fracionário, são considerados férmions. Pelo princício de exclusão de Pauli, os férmions não podem ocupar o mesmo estado quântico. Porém os pares de Cooper se comportam como bósons (partículas de spin inteiro) que podem se condensar num mesmo estado energético. Quando se estabelece este estado coletivo altamente ordenado, os superelétrons se movem sem que ocorra dissipação de energia.

A teoria BCS, apesar do sucesso em explicar de forma satisfatória o comportamento de supercondutores a baixas temperaturas, não tem o mesmo êxito em materiais de $T_{c}>35 K$. Em 1985, George Bednorz e Alex Müller [6] descobriram a supercondutividade em materiais cerâmicos, os quais possuem $T_{c}$ bem acima dos 
materiais BCS. As cerâmicas apresentam um comportamento muito diferente em relação aos metais, já que em sua maioria são maus condutores e os metais bons condutores.

A supercondutividade, mesmo sendo um fenômeno que ocorre a baixas temperaturas, tem sido utilizada em muitas aplicações práticas. Por exemplo, dispositivos, onde é necessário grande sensibilidade para se fazer medidas magnéticas (SQUID) ${ }^{2}$ e a confecção de eletroímãs capazes de gerar campos magnéticos muito elevados ${ }^{3}$ e consequentemente a possibilidade dos transportes de massa conhecidos como MAGLEV, trens que flutuam em poderosos eletroímãs na forma de trilhos.

\footnotetext{
${ }^{2}$ Dispositivo utilizado para medir campos magnéticos baixos.

${ }^{3}$ Para condutores normais a produção de eletroímãs poderosos é inviável.
} 


\section{Capítulo 2}

\section{Teorias Fenomenológicas da Supercondutividade}

\subsection{Introdução}

A revisão teórica feita neste Capítulo foi baseada predominantemente nos livros de Tinkham [11], Ketterson e Song [12] e Parks [13].

\subsection{O Modelo de London}

Os supercondutores basicamente apresentam três assinaturas: a condutividade perfeita, a exclusão total do campo magnético em seu interior e uma descontinuidade no calor específico do material (considerando o supercondutor na temperatura de transição $\left.T_{c}\right)$.

Com o objetivo de formular um modelo capaz de contemplar as características de um supercondutor (pelo menos tudo aquilo que se conhecia na época), London inicialmente fez um proposta considerando um condutor perfeito. Considerando um tratamento clássico, o desenvolvimento teórico se deu da seguinte forma. Considerando os portadores de carga submetidos apenas ao efeito de um campo elétrico E e fazendo uso da segunda lei de Newton podemos escrever a seguinte relação:

$$
m \dot{\mathbf{v}}=e \mathbf{E}
$$


onde $m$ é a massa dos portadores de carga (elétrons), $e$ a carga elétrica elementar, $\dot{\mathbf{v}}=d \mathbf{v} / d t$ é a aceleração. Introduzindo a densidade de corrente $\mathbf{J}=n e \mathbf{v}$, onde $n$ é a densidade de elétrons, podemos reescrever a equação acima da seguinte forma:

$$
\frac{d \mathbf{J}}{d t}=\frac{n e^{2}}{m} \mathbf{E}
$$

Esta é conhecida como a primeira equação de London.

Por outro lado, usando a lei de Ampère:

$$
\boldsymbol{\nabla} \times \mathbf{B}=\frac{4 \pi}{c} \mathbf{J}
$$

obtemos a seguinte equação para o campo magnético:

$$
\frac{\partial \boldsymbol{\nabla} \times \mathbf{B}}{\partial t}=\frac{4 \pi n e^{2}}{m c} \mathbf{E} .
$$

Agora, usando a lei de Faraday:

$$
\boldsymbol{\nabla} \times \mathbf{E}=-\frac{1}{c} \frac{\partial \mathbf{B}}{\partial t}
$$

e aplicando o rotacional a ambos os lados da equação (2.2), obtemos:

$$
\nabla \times \nabla \times \dot{\mathbf{B}}=-\frac{4 \pi n e^{2}}{m c^{2}} \dot{\mathbf{B}}
$$

podemos escrever: ${ }^{1}$

$$
\boldsymbol{\nabla} \times \boldsymbol{\nabla} \times \mathbf{B}+\frac{1}{\lambda^{2}} \mathbf{B}=0,
$$

onde $\lambda$ é um dos dois comprimentos fundamentais da supercondutividade conhecido como comprimento de penetração de London, e é definido por:

$$
\frac{1}{\lambda^{2}}=\frac{4 \pi n e^{2}}{m c^{2}}
$$

A equação (2.7) é conhecida como a segunda equação de London.

\footnotetext{
${ }^{1} \mathrm{O}$ lado direito da equação (2.7) é considerada nula. Isto será suficiente para descrever a penetração superficial do campo magnético. Na Seção 2.9, veremos que esta equação não é nula quando haver nucleação de vórtices no supercondutor.
} 
Alternativamente, os irmãos London fizeram uma proposta baseada num modelo de dois fluidos, onde um deles é um superfluido (ausente de viscosidade) e o outro, um fluido normal (com viscosidade finita). Eles propuseram que a energia livre pode ser dividida em três partes:

$$
F=F_{\mathrm{n}}+F_{\text {kin }}+F_{\text {mag }}
$$

onde $F_{\mathrm{n}}$ é a energia associada ao fluxo normal, $F_{\text {kin }}$ é a energia cinética associada ao movimento do superfluido e $F_{\text {mag }}$ é a energia do campo magnético.

Podemos escrever estas contribuições energéticas da seguinte forma:

$$
F_{\text {mag }}=\frac{1}{8 \pi} \int B^{2}(\mathbf{r}) d^{3} r
$$

e

$$
F_{\text {kin }}=\frac{1}{2} \int \rho(\mathbf{r}) v^{2} d^{3} r
$$

onde $\rho(\mathbf{r})$ é a densidade de massa associada ao superfluido.

Escrevendo $\rho=n m$ e $\mathbf{v}=(1 / n e) \mathbf{J}$ e usando a lei de Ampère (2.3), podemos reescrever a equação (2.11) na seguinte forma:

$$
F_{\text {kin }}=\frac{1}{8 \pi} \int \lambda^{2}(\boldsymbol{\nabla} \times \mathbf{B})^{2} d^{3} r
$$

onde $n$ agora é interpretado como densidade de super-elétrons. Somando todas as contribuições e usando cálculo variacional para minimizar a energia livre total, obtemos exatamente a segunda equação de London (2.7).

Iremos agora aplicar relação a um supercondutor semi-infinito. O supercondutor ocupa o espaço $x>0$ e o vácuo $x<0$. Considere um campo magnético externo aplicado $H$ paralelo ao plano $y z$, de tal forma que tenhamos $\mathbf{B}=\left(0,0, B_{z}(x)\right)$ no interior do supercondutor e $\mathbf{B}=H \mathbf{k}$ no vácuo. Tendo em vista estas simplificações, e usando a segunda equação de London, obtemos:

$$
\frac{d^{2} B_{z}}{d x^{2}}-\frac{1}{\lambda^{2}} B_{z}=0
$$


onde usamos a identidade vetorial $\boldsymbol{\nabla} \times(\boldsymbol{\nabla} \times \mathbf{B})=\boldsymbol{\nabla} \cdot(\boldsymbol{\nabla} \cdot \mathbf{B})-\boldsymbol{\nabla}^{2} \mathbf{B}$, e $\boldsymbol{\nabla} \cdot \mathbf{B}=0$.

Esta equação diferencial tem como solução:

$$
\mathbf{B}(x)=k H e^{-x / \lambda}
$$

Por meio deste resultado, notamos que o campo magnético paralelo à superfície do supercondutor penetra no material exponencialmente com um comprimento característico $\lambda$, conforme mostrado na Figura 2.1.

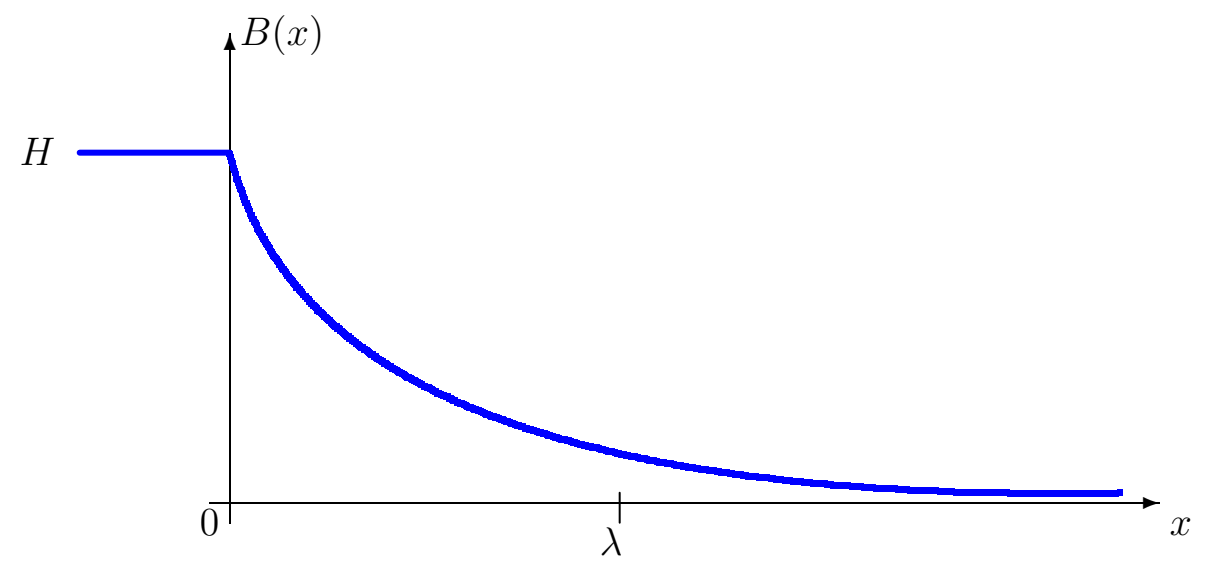

Figura 2.1: Comprimento característico de London.

Pela lei de Ampére, a densidade de corrente induzida é paralela ao plano yz e é dada por:

$$
\mathbf{J}(x)=-\mathbf{j} \frac{c}{4 \pi \lambda} H e^{-x / \lambda} .
$$

Esta é a densidade de corrente que impede o campo magnético de penetrar no interior do supercondutor; esta também é conhecida como corrente de blindagem ou circuitação.

Concluindo, podemos observar que as equações de London demonstram ser capazes de descrever o efeito Meissner e a penetração exponencial do campo magnético na superfície do supercondutor. 


\subsection{A Teoria de Ginzburg-Landau}

Em 1937, partindo de uma teoria já muito bem estabelecida, Landau desenvolveu um modelo para descrever transições de fase de segunda ordem . O ponto central da sua teoria foi a introdução de um parâmetro de ordem que desaparecia acima da temperatura crítica, $T>T_{c}$. A princípio, o parâmetro de ordem estudado foi a magnetização $\mathbf{M}$ que realmente apresentava o comportamento proposto em sua teoria, sendo o ponto de transição, conhecido como temperatura de Curie. De fato, a magnetização torna-se diferente de zero abaixo desta temperatura. Landau também percebeu que se o parâmetro de ordem desenvolve-se continuamente de zero até abaixo de $T_{c}$ é possível expandir a energia livre numa série de potências do parâmetro de ordem. Desprezando termos de ordens mais elevadas a expansão assume a forma:

$$
F=F_{0}+\alpha M^{2}+\frac{\beta}{2} M^{4}
$$

onde $F_{0}$ é a energia para a magnetização nula. A constante $\beta$ fornece a concavidade da curva gerada pela função acima. Se $\beta$ for negativo a energia será mínima para um valor arbitrariamente grande da magnetização, fato que sugere uma divergência. Portanto $\beta$ deve ser positivo para que tenhamos um valor finito de mínima energia. Já a constante $\alpha$, pode assumir qualquer sinal, uma vez que esta constante fornece apenas formas diferentes para a curva da função (2.16), porém não fazendo surgir nenhuma divergência.

Na Figura 2.2 exibimos esquematicamente a função (2.16). Assim, podemos concluir que:

- se $\alpha>0$, o mínimo ocorre em $M=0$;

- se $\alpha<0$, o mínimo ocorre em $M \neq 0$. 

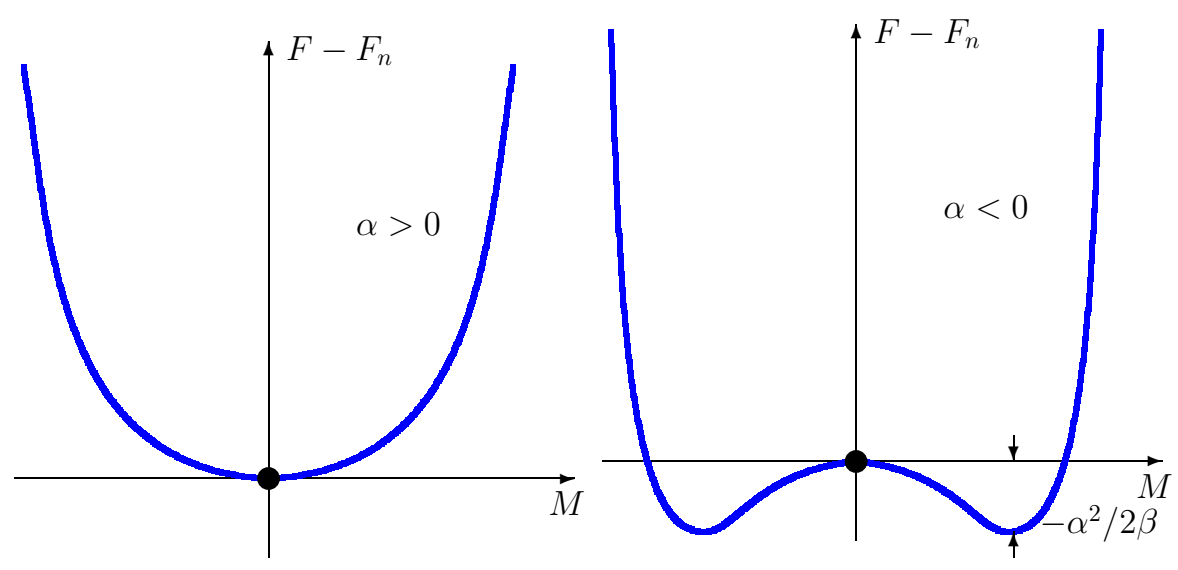

Figura 2.2: Funções de energia livre.

Para modelarmos um transição de fase de segunda ordem, devemos vincular o parâmetro de ordem com a temperatura. Como $\alpha$ deve ser negativo, para que assim tenhamos um mínimo de magnetização diferente de zero, podemos estabelecer a seguinte relação: $\alpha(T)=a\left(T-T_{c}\right)$ para $T \leq T_{c}$ e $\alpha(T)=0$ para $T>T_{c}$, pois desta maneira, $\alpha$ troca de sinal em $T_{c}$

Analisando o gráfico acima podemos encontrar os mínimos para a energia em relação a $M$, por um processo bastante simples:

$$
\frac{\partial F}{\partial M}=0 \Rightarrow\left[\alpha(T)+\beta M^{2}\right] M=0
$$

Esta equação apresenta duas soluções:

$$
\begin{array}{lll}
\alpha>0 \Rightarrow M=0 & \text { para } T>T_{c} \\
\alpha \leq 0 \Rightarrow M^{2}=-\frac{\alpha}{\beta}=-\frac{a\left(T-T_{c}\right)}{\beta} & \text { para } T \leq T_{c} .
\end{array}
$$

De fato, com a relação (2.18), percebemos que, com o aumento da temperatura (abaixo de $T_{c}$ ), o módulo da magnetização vai diminuindo até anular-se em $T=T_{c}$.

A supercondutividade e a magnetização são tipos de estados macroscópicos, e além disso esses estados apresentam um comportamento equivalente no que diz respeito à transição de fase, ou seja, existe também um ponto em que o material transiciona da fase normal para a supercondutora e vice-versa. 
Ginzburg e Landau empregaram esta mesma idéia da teoria de transição de fase de segunda ordem de Landau na supercondutividade, assumindo a existência de uma função de onda macroscópica, $\psi$, a qual eles tomaram como o parâmetro de ordem associado à supercondutividade. Desde que a função de onda possa ser complexa, apenas a forma $\psi \bar{\psi}$ pode entrar na expansão da energia livre. Portanto, podemos escrever:

$$
F=F_{0}+\alpha|\psi|^{2}+\frac{\beta}{2}|\psi|^{4}
$$

A minimização energética prossegue exatamente como a que foi feita para a magnetização, já que são quantidades físicas que podem ser tratadas de forma equivalente:

$$
\begin{array}{ll}
|\psi|=0, & \text { para } T>T_{c}, \\
|\psi|=\left[\frac{a\left(T_{c}-T\right)}{\beta}\right]^{1 / 2}, & \text { para } T \leq T_{c} .
\end{array}
$$

Como o estado supercondutor é inomogêneo, interpretaremos $F$ como energia livre, podendo ser escrita da seguinte forma:

$$
F=F_{0}+\int \mathscr{F}_{C}[\psi(\mathbf{r})] d^{3} r
$$

onde

$$
\mathscr{F}_{C}=\alpha|\psi|^{2}+\frac{\beta}{2}|\psi|^{4}
$$

e $F_{0}$ é a energia correspondente ao estado normal.

A relação acima não refletia o aumento da energia associado com uma distorção espacial do parâmetro de ordem (efeitos associados ao comprimento de coerência, um parâmetro muito importante que veremos mais adiante). Para dar conta de tais efeitos Ginzburg e Landau acrescentaram um termo que seria um gradiente do parâmetro de ordem. Temos que:

$$
\mathscr{F}_{G}=\frac{\hbar^{2}}{2 m^{*}}|\nabla \psi|^{2}
$$

onde $m^{*}$ sendo um parâmetro e a escolha do coeficiente $\hbar^{2} / 2 m^{*}$ se assemelha à energia cinética na mecânica quântica. 
Ginzburg e Landau assumiram que a relação acima era a contribuição da energia cinética dos super-elétrons.

Na presença de campo magnético é necessário então a introdução do momento linear cinético, que tem a seguinte forma:

$$
\mathbf{p}=-i \hbar \nabla-\frac{e^{*}}{c} \mathbf{A} .
$$

Substituindo (2.24) em (2.23) obtemos:

$$
\mathscr{F}_{G}=\frac{\hbar^{2}}{2 m^{*}}\left|\left[\boldsymbol{\nabla}-\frac{i e^{*}}{\hbar c} \mathbf{A}(\mathbf{r})\right] \psi\right|^{2} .
$$

Finalmente, considerando a contribuição do campo magnético para a densidade de energia que pode ser escrita da seguinte forma:

$$
\mathscr{F}_{B}=\frac{1}{8 \pi} B^{2}(\mathbf{r}),
$$

podemos escrever a energia total como:

$$
\begin{aligned}
F & =F_{0}+\int\left(\mathscr{F}_{C}+\mathscr{F}_{G}+\mathscr{F}_{H}\right) d^{3} r \\
& =F_{0}+\int\left\{\alpha|\psi|^{2}+\frac{\beta}{2}|\psi|^{4}+\frac{\hbar^{2}}{2 m^{*}}\left|\left[\nabla-\frac{i e^{*}}{\hbar c} \mathbf{A}\right] \psi\right|^{2}+\frac{1}{8 \pi} B^{2}\right\} d^{3} r .
\end{aligned}
$$

As funções $\psi$ e $\mathbf{A}$ as quais minimizam o funcional energia livre, podem ser obtidas através das equações de Euler-Lagrange:

$$
\begin{aligned}
\frac{\partial \mathscr{F}}{\partial \psi}-\boldsymbol{\nabla} \cdot\left[\frac{\partial \mathscr{F}}{\partial(\boldsymbol{\nabla} \psi)}\right] & =0, \\
\frac{\partial \mathscr{F}}{\partial \mathbf{A}}-\boldsymbol{\nabla} \times\left[\frac{\partial \mathscr{F}}{\partial(\boldsymbol{\nabla} \times \mathbf{A})}\right] & =0 .
\end{aligned}
$$

Desta forma obtemos as equações de Ginzburg-Landau:

$$
\begin{gathered}
-\frac{\hbar^{2}}{2 m^{*}}\left[\boldsymbol{\nabla}-\frac{i e^{*}}{\hbar c} \mathbf{A}\right]^{2} \psi+\alpha \psi+\beta|\psi|^{2} \psi=0 \\
\boldsymbol{\nabla} \times \boldsymbol{\nabla} \times \mathbf{A}=\frac{e *}{2 m^{*}}\left\{\bar{\psi}\left[-i \hbar \boldsymbol{\nabla}-\frac{e^{*}}{c} \mathbf{A}\right] \psi+\psi\left[+i \hbar \boldsymbol{\nabla}-\frac{e^{*}}{c} \mathbf{A}\right] \bar{\psi}\right\} .
\end{gathered}
$$


A segunda das equações de Ginzburg-Landau nada mais é do que a lei de Ampère:

$$
\nabla \times \mathbf{B}=\frac{4 \pi}{c} \mathbf{J}
$$

onde

$$
\mathbf{J}=\frac{-i \hbar e^{*}}{2 m^{*}}[\bar{\psi} \boldsymbol{\nabla} \psi-\psi \boldsymbol{\nabla} \bar{\psi}]-\frac{\left(e^{*}\right)^{2}}{m^{*} c}|\psi|^{2} \mathbf{A} .
$$

A relação acima é conhecida como densidade de corrente supercondutora.

\subsection{Comprimentos Característicos}

\subsubsection{Comprimento de Coerência}

Primeiramente examinaremos o caso simples envolvendo uma inomogeneidade do parâmetro de ordem gerada pela presença de um contorno, na ausência de um campo magnético. Considerando apenas a direção $x$ e que a fronteira se encontra exatamente no plano yz, a primeira equação de Ginzburg-Landau toma a seguinte forma:

$$
-\frac{\hbar^{2}}{2 m^{*}} \frac{d^{2} \psi}{d x^{2}}+\alpha \psi+\beta \psi^{3}=0
$$

No estado supercondutor, $\alpha$ é negativo. Podemos escrever $\alpha=-|\alpha|$. Substituindo na equação acima teremos:

$$
-\frac{\hbar}{2 m^{*}} \frac{d^{2} \psi}{d x^{2}}-|\alpha| \psi+\beta \psi^{3}=0 .
$$

Neste momento é conveniente escrevermos:

$$
\psi=\sqrt{\frac{|\alpha|}{\beta}} f,
$$

de onde obtemos que:

$$
\xi^{2} \frac{d^{2} f}{d x^{2}}-f+f^{3}=0 .
$$

O coeficiente da segunda derivada tem unidades de quadrado de comprimento e é definido por:

$$
\xi^{2}=\frac{\hbar^{2}}{2 m^{*}|\alpha|},
$$


o qual é conhecido como comprimento de coerência. Veremos mais adiante que $\xi$ é o comprimento ao longo do qual temos uma variação significativa do parâmetro de ordem.

Em nossa análise é importante lembrar que estamos trabalhando com um supercondutor semi-infinito, conforme descrito no início desta seção. Portanto, podemos admitir como condição de contorno que, para $x \rightarrow \infty$, temos $f^{2}=1$, pois num ponto longe da fronteira a amostra é totalmente supercondutora. Também podemos considerar que $f^{\prime}=0$ para $x \rightarrow \infty$. Aqui usamos a notação $f^{\prime}=d f / d x$.

Multiplicando a equação (2.36) por $f^{\prime}$, e integrando o resultado e em seguida derivando em relação a $x$, obtemos:

$$
\frac{d}{d x}\left[\frac{-\xi^{2}\left(f^{\prime}\right)^{2}}{2}-\frac{f^{2}}{2}+\frac{f^{4}}{4}\right]=0
$$

de onde encontramos que:

$$
\frac{-\xi^{2}\left(f^{\prime}\right)^{2}}{2}-\frac{f^{2}}{2}+\frac{f^{4}}{4}=C .
$$

Agora, utilizando as condições de contorno $f^{2}=1$ e $f^{\prime}=0$ para $x \rightarrow \infty$, determinamos o valor da constante $C=1 / 4$. Em seguida, substituindo o valor de $C$, encontramos:

$$
\xi^{2}\left(f^{\prime}\right)^{2}=\frac{1}{2}\left(1-f^{2}\right)^{2}
$$

Esta equação pode facilmente ser resolvida por meio de métodos elementares de integração. Encontramos:

$$
f=\operatorname{arctanh}\left(\frac{x}{\sqrt{2} \xi}\right) .
$$

A Figura 2.3 ilustra o comportamento desta função. Conforme antecipamos acima, o parâmetro de ordem varia ao longo da distância $\xi$.

Uma vez que $\alpha(T)=a\left(T-T_{c}\right)$, ou ainda, $\alpha(T)=-a T_{c}\left[1-\left(T / T_{c}\right)\right]$, substituindo na relação do comprimento de coerência (2.37), obtemos uma relação dependente 


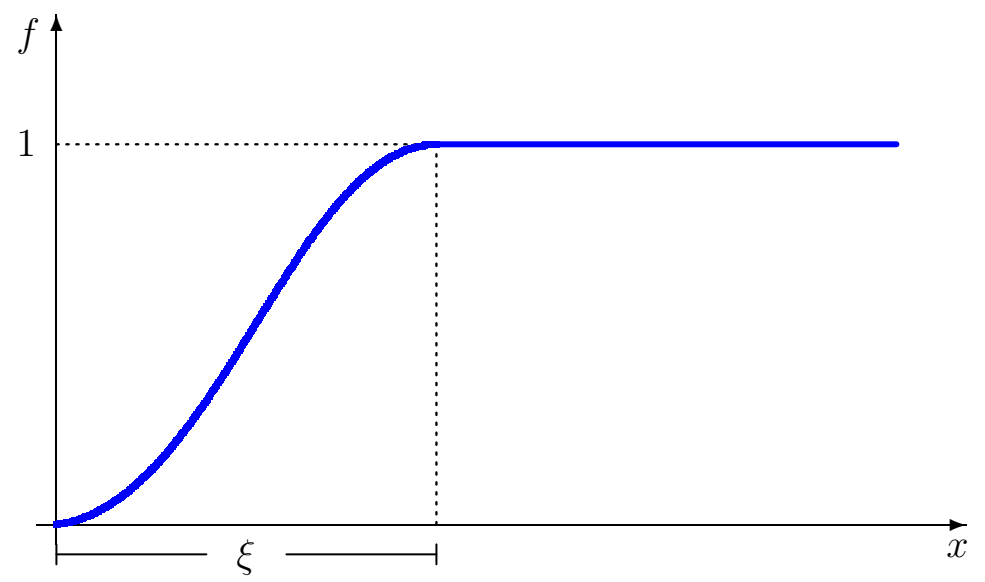

Figura 2.3: Esboço do comportamento do parâmetro de ordem na interface vácuosupercondutor.

da temperatura:

$$
\xi(T)=\sqrt{\frac{\hbar^{2}}{2 m^{*} a T_{c}}}\left(1-\frac{T}{T_{c}}\right)^{-1 / 2}=\xi(0)\left(1-\frac{T}{T_{c}}\right)^{-1 / 2} .
$$

\subsubsection{Comprimento de Penetração de London}

Em segundo lugar, consideramos a situação distinta da anterior onde o supercondutor encontra-se na presença de um campo magnético aplicado. Consideramos regiões onde o parâmetro de ordem praticamente é uma constante e é dado por $n=|\psi|^{2}=|\alpha| / \beta$. A densidade de corrente supercondutora (2.32) assume a forma:

$$
\mathbf{J}=-\frac{\left(e^{*}\right)^{2}}{m^{*} c}|\psi|^{2} \mathbf{A}
$$

Substituindo esta equação na lei de Ampère (2.31), vem que:

$$
\frac{m^{*} c^{2} \beta}{4 \pi\left(e^{*}\right)^{2}|\alpha|} \boldsymbol{\nabla} \times \mathbf{B}+\mathbf{A}=0
$$

Aplicando o rotacional a ambos os lados da equação acima obtemos a equação de London:

$$
\lambda^{2} \boldsymbol{\nabla} \times \boldsymbol{\nabla} \times \mathbf{B}+\mathbf{B}=0,
$$


onde agora o comprimento de penetração de London dependente da temperatura e é dado por:

$$
\lambda^{2}=\frac{m^{*} c^{2} \beta}{4 \pi\left(e^{*}\right)^{2}|\alpha|},
$$

ou ainda, substituindo a constante fenomenológica $\alpha(T)=-a T_{c}\left[1-\left(T / T_{c}\right)\right]$, temos que:

$$
\lambda^{2}=\frac{m^{*} c^{2} \beta}{4 \pi\left(e^{*}\right)^{2} a T_{c}}\left(1-\frac{T}{T_{c}}\right)^{-1}=\lambda^{2}(0)\left(1-\frac{T}{T_{c}}\right)^{-1} .
$$

Neste momento introduziremos um parâmetro, o qual é dado pela razão entre $\lambda$ e $\xi$ :

$$
\kappa=\frac{\lambda(T)}{\xi(T)}=\frac{m^{*} c}{e^{*} \hbar} \sqrt{\frac{\beta}{2 \pi}} .
$$

Embora os comprimentos fundamentais dependam da temperatura, $\kappa$ não depende; $\kappa$ é conhecido como parâmetro de Ginzburg-Landau. Conforme veremos mais adiante, este parâmetro caracteriza o material supercondutor.

Gorkov mostrou que as equações de Ginzburg-Landau podem ser obtidas a partir de considerações puramente microscópicas para $T \approx T_{c}$, ou seja, que a teoria fenomenológica emerge naturalmemte da teoria BCS a partir de primeiros princípios. Muito embora a teoria de Ginzburg-Landau foi concebida para ser válida somente próximo da temperatura crítica, ela também vem sendo aplicada satisfatoriamente para intervalos de temperaturas bem abaixo de $T_{c}$.

\subsection{Campos Críticos}

Em 1957, Alexei Abrikosov sugeriu que os supercondutores se comportam de forma bastante distinta quando submetidos a campos magnéticos. Ele chamou de supercondutor do tipo-I aquele que, quando na presença de um campo magnético aplicado, sofre uma transição de primeira ordem num ponto de descontinuidade da magnetização, conhecido como campo crítico $H_{c}(T)$; a partir deste valor de campo magnético, a supercondutividade é totalmente suprimida. Ele também propôs que pode existir 
uma outra classe de materiais, que chamou de supercondutores do tipo-II, os quais exibem uma penetração parcial de fluxo magnético, começando num campo crítico inferior $H_{c 1}(T)$ até atingir um campo crítico superior $H_{c 2}(T)$, valor a partir do qual o material volta ao estado normal. Esta entrada parcial de fluxo magnético ocorre de forma quantizada em unidades de quantum de fluxo $\Phi_{0}=h c / 2 e$ penetrando no supercondutor na forma de vórtices (na Seção 2.9 descrevemos em mais detalhes a estrutura de um vórtice). Esta condição de penetração parcial é chamada de estado misto, onde há uma coexistência de regiões supercondutoras e normais; no núcleo de um vórtice a supercondutividade é totalmente destruída e ao seu redor ainda temos a presença de supercorrentes. Nos supercondutores do tipo-II, devido a essa penetração parcial de fluxo magnético, a energia do estado diamagnético, para manter o campo magnético no exterior do supercondutor, é menor. Então, $H_{c 2}(T)$ pode ser muito maior que o campo crítico $H_{c}(T)$. Essa propriedade permite que materiais supercondutores do tipo-II podem suportar campos magnéticos bem mais elevados que os materiais do tipo-I.

Existe ainda um terceiro campo crítico chamado $H_{c 3}(T)$. Como veremos mais adiante, os vórtices auto-organizam-se em forma de uma rede periódica. Tanto mais próximo de $H_{c 2}(T)$, maior a densidade de vórtices. Quando $H$ alcança o valor do segundo campo crítico, a rede é inteiramente destruída, porém restando supercondutividade na superfície. Continuando a aumentar $H$, quando atigimos um determinado valor $H_{c 3}(T)$, a supercondutividade superficial é destruída.

\subsection{Classificação dos Supercondutores}

O valor do parâmetro de Ginzburg-Landau $\kappa$ introduzido na Seção 2.4.2, diferencia os supercondutores em tipo-I e tipo-II, conforme mencionamos anteriormente. 
A energia de superfície (grandeza física semelhante à tensão superficial) ${ }^{2}$ pode ser descrita por meio do seguinte parâmetro: [14]

$$
\gamma=\frac{|\alpha|^{2}}{2 \beta} \lambda \int_{0}^{\infty}\left\{\left(1-|\psi|^{4}\right)-\left[1-(B-1)^{2}\right]\right\} d x .
$$

Na equação acima o campo está em unidades de $\sqrt{2} H_{c}$ e os comprimentos em unidades de $\lambda$.

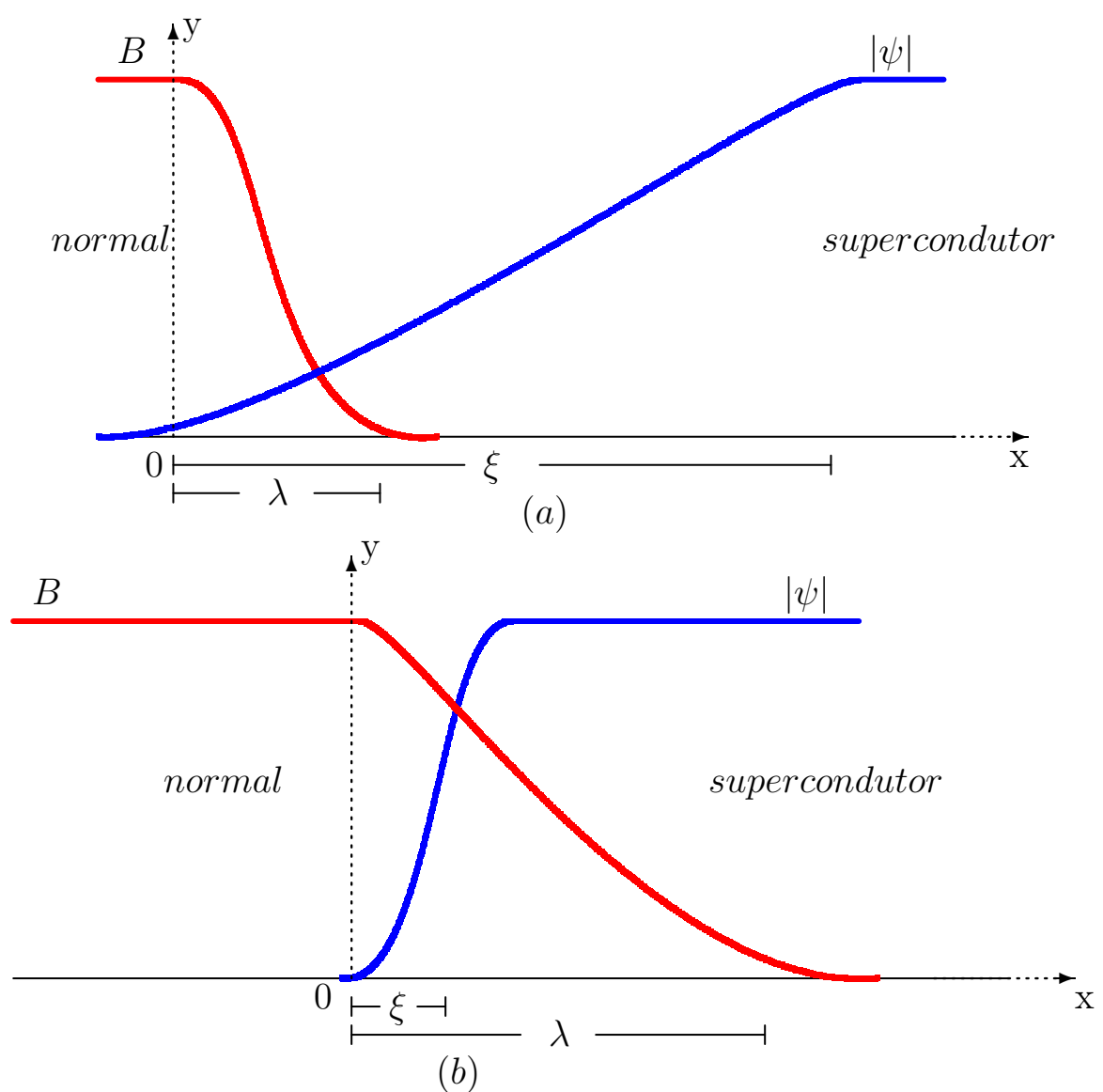

Figura 2.4: Distribuição espacial do parâmetro de ordem $\psi$ e do campo magnético B numa interface supercondutor-normal. Os paineis (a) e (b) são referentes a supercondutores do tipo-I e II, respectivamente.

Uma estimativa do valor de $\gamma$ pode ser obtida usando as seguintes aproximações

\footnotetext{
${ }^{2} \mathrm{O}$ parâmetro $\gamma$ pode ser obtido tomando a diferença de energia de Gibbs entre dois meios: um normal e um supercondutor. O campo magnético é aplicado na região normal e paralelo à superfície supercondutora.
} 
(ver Figura 2.4):

$$
|\psi|= \begin{cases}1, & x>\xi \\ 0, & x<\xi\end{cases}
$$

e

$$
B= \begin{cases}1, & x<\lambda \\ 0, & x>\lambda\end{cases}
$$

Portanto, a equação (2.49) assume a forma:

$$
\gamma \simeq \frac{|\alpha|^{2}}{2 \beta} \lambda\left[\int_{0}^{1 / \kappa} d x-\int_{0}^{1} d x\right]
$$

de onde resulta que:

$$
\gamma \simeq \frac{|\alpha|^{2}}{2 \beta}(\xi-\lambda)
$$

Observando mais uma vez a Figura 2.4 é possível concluir por intermédio da equação (2.53) que a energia de um supercondutor do tipo-I é positiva e do tipo-II é negativa, o que nos leva a escrever:

- Tipo-I, $\xi>\lambda$,

- Tipo-II, $\xi<\lambda$.

Entretanto é possível obter um valor exato para $\gamma$ o qual é dado por:

$$
\gamma=\frac{|\alpha|^{2}}{2 \beta}\left(\frac{1}{\kappa}-\sqrt{2}\right)
$$

o que nos leva à seguinte definição exata:

- Tipo-I, $\kappa<\frac{1}{\sqrt{2}}$,

- Tipo-II, $\kappa>\frac{1}{\sqrt{2}}$. 

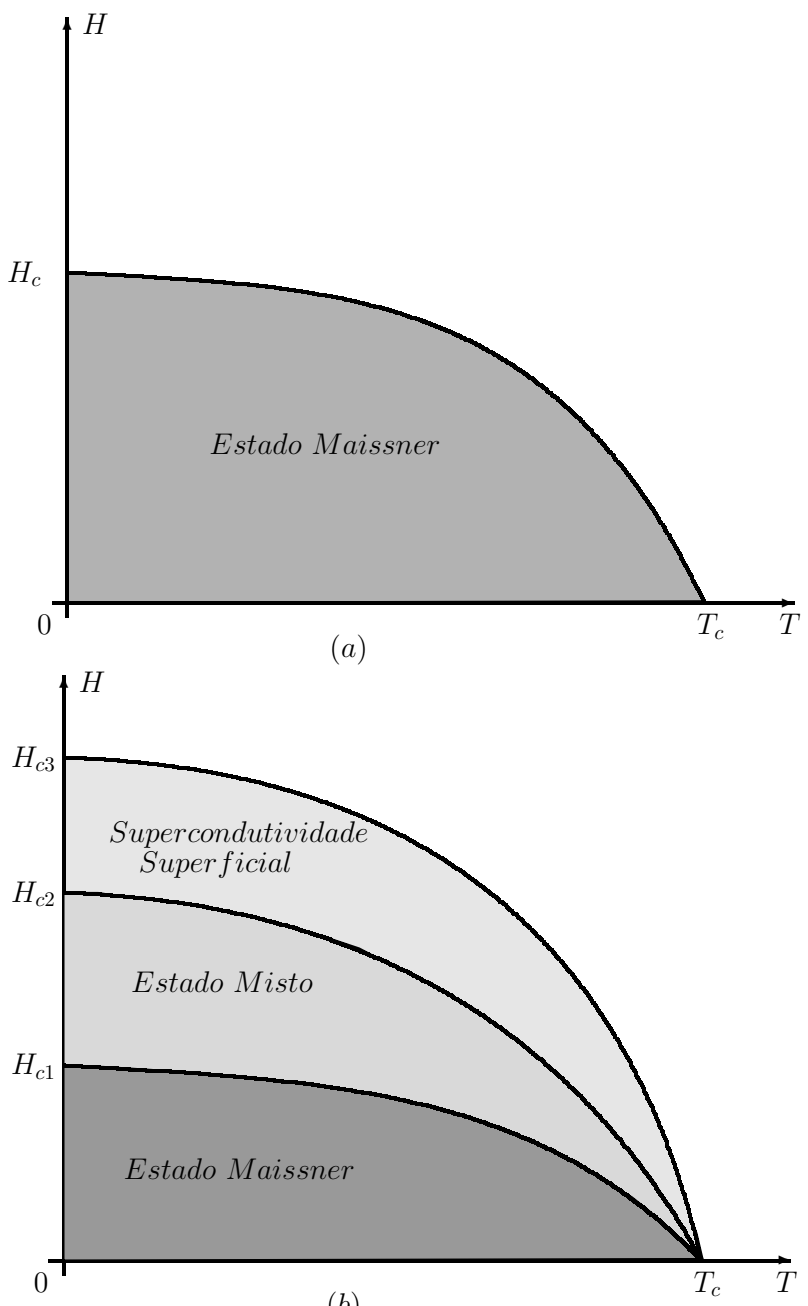

(b)

Figura 2.5: Diagrama de fase H-T para supercondutores do tipo-I (a) e tipo-II (b).

\subsection{Diagrama de Fase}

Os campos críticos $H_{c}(T), H_{c 1}(T), H_{c 2}(T)$ e $H_{c 3}(T)$ dependem da temperatura. A seguir ilustramos os diagramas de fase (ver Figura 2.5) que mostram a diferença entre supercondutores do tipo-I e do tipo-II em relação aos campos críticos.

No diagrama (a) da Figura 2.5 nota-se a existência de apenas um campo crítico $H_{c}(T)$, que separa o estado supercondutor (estado Meissner) do estado normal. Já o diagrama (b) apresenta três campos críticos. Observa-se que abaixo do campo crítico $H_{c 1}(T)$ temos o estado Meissner completo. Acima do campo $H_{c 1}(T)$ e abaixo do 
campo crítico $H_{c 2}(T)$, temos o estado misto. E finalmente acima deste último campo $H_{c 2}(T)$ e abaixo do campo $H_{c 3}(T)$, temos uma supercondutividade superficial. Uma vez superado o campo $H_{c 3}(T)$, o estado normal é alcançado.

Uma quantidade física importante no estudo da supercondutividade é a magnetização, pois ela é mensurável. A magnetização é definida como $\mathbf{M}=(\overline{\mathbf{B}}-\mathbf{H}) / 4 \pi$, onde $\overline{\mathbf{B}}$ é uma média espacial do campo magnético local $\mathbf{B}$. Num supercondudor do tipo-I, abaixo de $H_{c}(T)$ o campo é totalmente expelido da amostra. Então temos, $\mathbf{B}=0$ e $-4 \pi \mathbf{M}=\mathbf{H}$. Acima de $H_{c}(T)$ o campo magnético penetra em todo o supercondutor, $\mathbf{B}=\mathbf{H}$ e $\mathbf{M}=0$. Por outro lado, para supercondutores do tipo-II, acima do primeiro campo crítico, ao invés de anular-se totalmente, a magnetização decresce monotonicamente até desaparecer no segundo campo crítico $H_{c 2}(T)$. Esse comportamento da magnetização pode ser melhor compreendido através da Figura 2.6 .
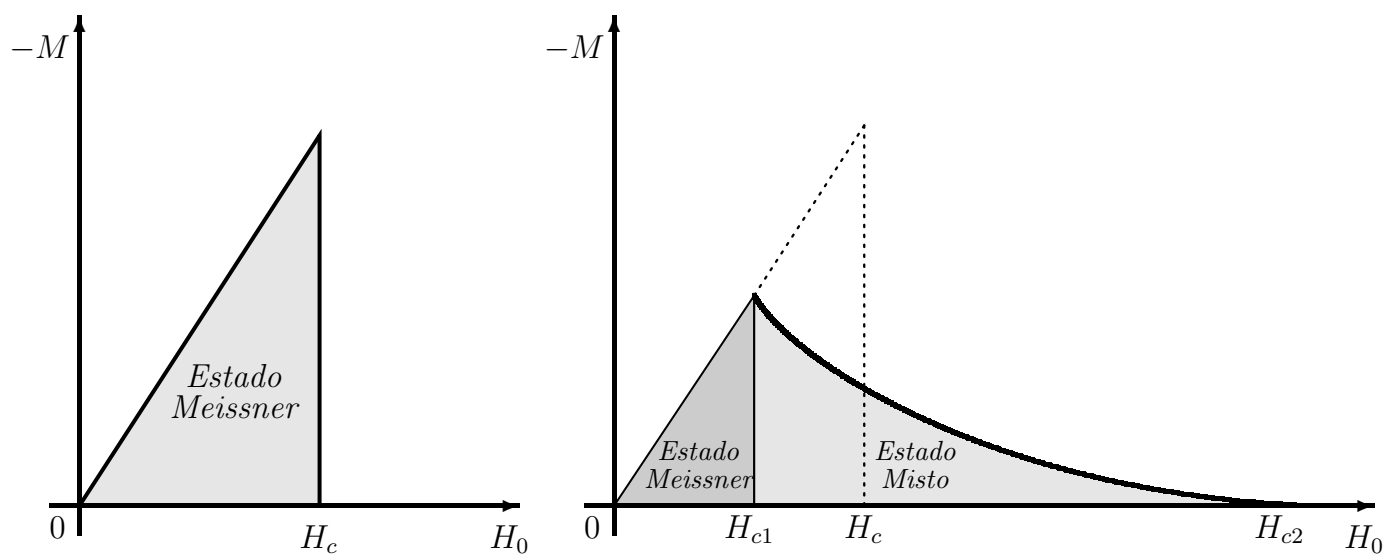

Figura 2.6: Magnetização em função do campo aplicado: supercondutor do tipo-I (esquerda), supercondutor do tipo-II (direita).

\subsection{Quantização de fluxo}

A quantização de fluxo garante que o fluxo no interior da amostra é sempre um múltiplo inteiro de um quantum de fluxo. Nesta Seção, apresentaremos uma justificativa 
da quantização do fluxo magnético no interior de supercondutores do tipo-II. Partiremos da equação (2.32) substituindo o parâmetro de ordem na forma $\psi=|\psi| e^{i \phi}$. Temos que:

$$
\mathbf{J}=\frac{2 e \hbar}{m^{*}}|\psi|^{2} \boldsymbol{\nabla} \phi-\frac{4 e^{2}}{m^{*} c} \mathbf{A}|\psi|^{2}
$$

Esta equação também pode ser reescrita na forma:

$$
\mathbf{A}=\frac{\hbar c}{2 e} \boldsymbol{\nabla} \phi-\frac{m^{*} c \mathbf{J}}{4 e^{2}|\psi|^{2}}
$$

Agora, usando o teorema de Stokes, vem que:

$$
\oint_{C} \mathbf{A} \cdot d \mathbf{r}=\int_{S} \boldsymbol{\nabla} \times \mathbf{A} \cdot \mathbf{n} d S=\int_{S} \mathbf{B} \cdot \mathbf{n} d S=\Phi,
$$

onde $S$ é uma superfície bilateral restrita ao caminho fechado $C$. Assim, integrando o potencial vetor da equação (2.56), encontramos:

$$
\frac{\hbar c}{2 e} \oint_{C} \nabla \phi \cdot d \mathbf{r}-\frac{m^{*} c}{4 e^{2}} \oint_{C} \frac{\mathbf{J}}{|\psi|^{2}} \cdot d \mathbf{r}=\Phi
$$

Em uma volta completa em torno do centro do vórtice, a fase varia de $2 \pi$; admitindo a existência de $n$ quanta, encerrados pela trajetória $C$, teríamos uma variação de $2 \pi n .^{3}$ Temos que:

$$
\Phi=n \frac{h c}{2 e}-\frac{m^{*} c}{4 e^{2}} \int_{C} \frac{\mathbf{J}}{|\psi|^{2}} \cdot d \mathbf{r}
$$

Para supercondutores macroscópicos, de dimensões muito maiores que o comprimento de penetração $\lambda$, podemos considerar $\mathbf{J}=0$ ao longo de $C$. Assim, temos que:

$$
\Phi=n \frac{h c}{2 e}=n \Phi_{0}
$$

onde $n$ é um número inteiro e conhecido como número quântico de fluxóide: determina o número de quanta de fluxo $\Phi_{0}$ que atravessa uma determinada área de seção reta do material supercondutor. A equação (2.60) mostra que o fluxo confinado no supercondutor é quantizado.

\footnotetext{
${ }^{3}$ Esta condição é conhecida como quantização de Bohr-Sommerfeld.
} 


\subsection{Estrutura de um Vórtice}

O vórtice tem um estrutura filamentar como em um tubo cilíndrico. No núcleo do vórtice, em uma extensão equivalente ao comprimento de coerência, o parâmetro de ordem decresce monotonicamente anulando-se no centro. Por outro lado, no centro do vórtice o campo magnético tem valor máximo e decresce monotonicamente na medida em que nos afastamos do centro. A dimensão de um vórtice é equivalente ao comprimento de penetração de London, conforme ilustrado esquematicamente na Figura 2.7. As supercorrentes anulam-se no núcleo do vórtice e persistem ao seu redor, blindando a região normal.

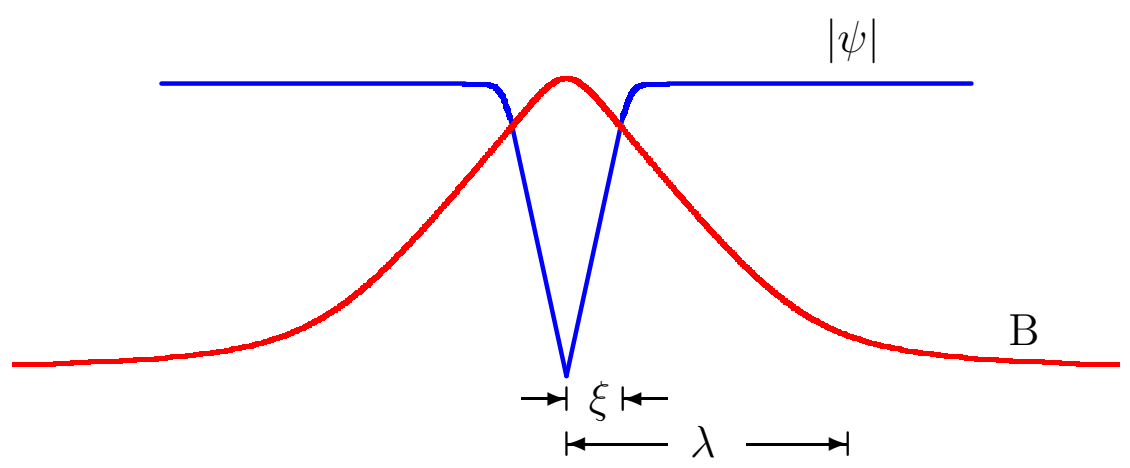

Figura 2.7: Estrutura de um vórtice.

Uma estimativa da forma do campo magnético em torno de um vórtice pode ser feita a partir do limite de London. Nesse limite $\xi \ll \lambda$, onde pode-se considerar que $|\psi|^{2}$ é constante em todo o espaço, exceto no núcleo do vórtice. No entanto, consideraremos a variação espacial da fase. Da lei de Ampère (2.31) e da expressão da densidade de corrente supercondutora (2.32), e usando a condição topológica da fase:

$$
\nabla \times \nabla \phi=\Phi_{0} \delta(\mathbf{r}),
$$

obtemos a equação de London:

$$
-\lambda^{2} \nabla^{2} \mathbf{B}+\mathbf{B}=\mathbf{k} \Phi_{0} \delta(\mathbf{r}) .
$$


A solução desta equação pode ser determinada usando a técnica de transformada de Fourier. Temos que:

$$
\mathbf{B}=\mathbf{k} \frac{\Phi_{0}}{2 \pi \lambda^{2}} K_{0}(r / \lambda)
$$

onde $K_{0}(x)$ é função de Bessel $[15,16]$ modificada de ordem zero.

A função de Bessel modificada tem os seguintes limites assimptóticos:

- para $r \gg \lambda$ a função $K_{0}(r / \lambda)$ decai exponencialmente na forma $\sqrt{\pi \lambda / 2 r} e^{-r / \lambda}$,

- para $r \ll \lambda$ a função $K_{0}(r / \lambda)$ diverge logaritmicamente na forma $\ln (\lambda / r)$.

Assim, pelo primeiro item, podemos observar que a interação entre vórtices é de curto alcance, uma vez que decresce exponencialmente. Pelo segundo item, notamos que na escala de baixos comprimentos o campo magnético diverge logaritmicamente. Esta divergência é artificial, pois desprezamos as dimensões do caroço do vórtice tomando $|\psi|^{2}$ constante em todo o espaço.

\subsection{Vórtices em Supercondutores Macroscópicos}

Usando as equações de Ginzburg-Landau, em 1957 Abrikosov propôs que no estado misto, entre os campos $H_{c 1}(T)$ e $H_{c 2}(T)$, o fluxo não penetra no supercondutor em um único domínio. Ele penetra num arranjo de tubos de fluxo, onde cada um deles carrega um quantum de fluxo:

$$
\Phi_{0}=\frac{h c}{2 e}=2.07 \times 10^{-7} \mathrm{G} \cdot \mathrm{cm}^{2} .
$$

Este arranjo periódico de vórtices encontra-se ilustrado na Figura 2.8. Em cada célula unitária do arranjo, temos um quantum de fluxo. Abrikosov estudou dois arranjos, um quadrado e outro triangular.

Ele previa que na região de altos campos do diagrama de fase, a rede de vórtices era quadrada e que para baixo campo ela se tornava triangular. Assim, ele acreditava 


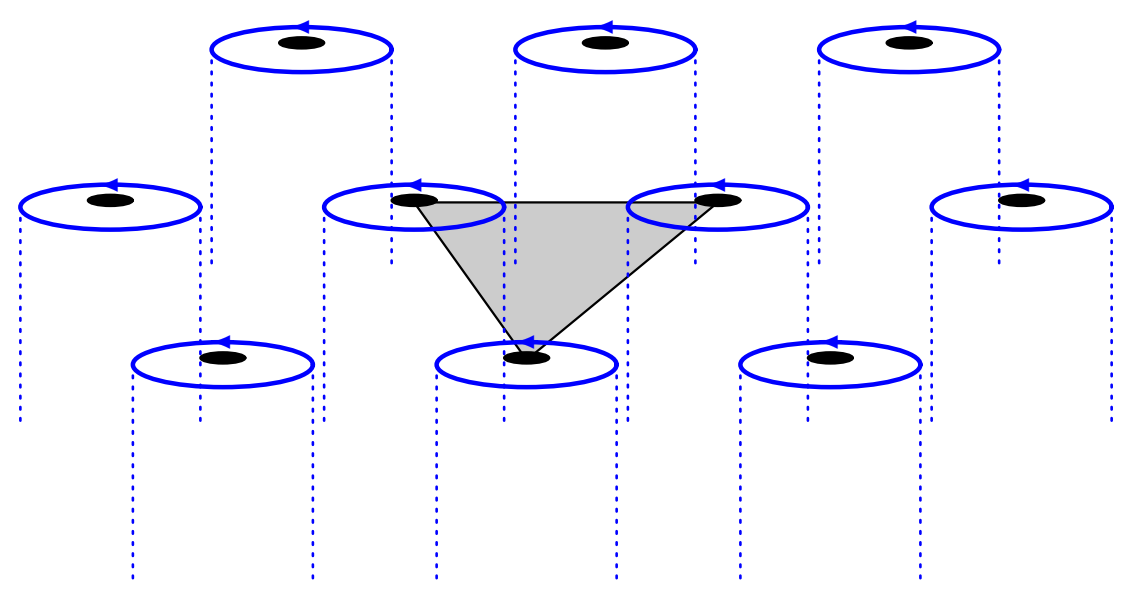

Figura 2.8: Rede de vórtices de Abrikosov.

que poderia haver uma transição de fase estrutural dentro do estado misto. Porém, posteriormente, foi mostrado que o arranjo numa forma triangular (ver Figura 2.8) seria o mais estável em todo o diagrama de fase. Esta rede de maior estabilidade (a triangular) é conhecida como rede de Abrikosov. A rede triangular de vórtices foi observada mais tarde por técnicas experimentais de decoração magnética com o auxílio de microscopia eletrônica.[9]

\subsection{Supercondutividade Mesoscópica}

Com o advento da nanotecnologia, tornou-se possível a fabricação de amostras com dimensões cada vez menores num processo conhecido como microfabricação. Desta forma, é possível a fabricação de supercondutores com dimensões da ordem dos comprimentos característicos de penetração de London $\lambda$ e de coerência $\xi$. Para supercondutores convencionais, na temperatura zero, podemos citar alguns exemplos:

- Pb-In: $\lambda=150 n m, \xi=30 n m$;

- Pb-Bi: $\lambda=200 n m, \xi=20 n m$;

- Nb-Ti: $\lambda=300 n m, \xi=4 n m$. 
Amostras com essas dimensões são conhecidas como amostras mesoscópicas, onde as propriedades supercondutoras são influenciadas consideravelmente pelos efeitos de confinamento. Na supercondutividade a configuração de vórtices depende fortemente das condições de contorno e também da geometria da amostra. Isso nos leva a concluir que amostras mesoscópicas apresentam comportamentos diferentes em relação a amostras macroscópicas. Por isso, em se tratando de filmes supercondutores de espessura comparável aos comprimentos característicos, a diferenciação de supercondutores do tipo-I e do tipo-II, não depende somente do parâmetro de Ginzburg-Landau $\kappa$, mas também da espessura da amostra. A espessura pode ser relacionada com o comprimento de penetração de London da seguinte forma:

$$
\Lambda=\frac{\lambda^{2}}{d}
$$

onde $\Lambda$ é conhecido com o comprimento de penetração efetivo. [17]

Com isso, dependendo da espessura do filme, podemos ter um estado misto mesmo em supercondutores do tipo-I em que $\kappa^{*}=\Lambda / d>1 / \sqrt{2}$.

A maneira com que o fluxo penetra num supercondutor mesoscópico do tipo-II, depende fortemente da forma, do tamanho, do campo externo aplicado e da temperatura da amostra como sugerem alguns resultados experimentais e teóricos recentes. [18-21] A rede de vórtices nestas geometrias confinadas diferem substancialmente da rede de Abrikosov. Podemos ter duas configurações de estado de vórtices: os multivórtices (vórtices isolados), cada qual com vorticidade um, carregando um quantum de fluxo, ou ainda vórtices gigantes, carregando uma vorticidade superior a um. $\mathrm{Na}$ Figura 2.9 exibimos estes dois estados para o caso de vorticidade total 3.

Nas regiões de cores vermelhas temos alta densidade de pares de Cooper, ou seja, regiões onde a amostra é fortemente supercondutora. Por outro lado, nas regiões azuis (com preto no centro) temos a amostra no estado normal, onde o campo magnético local é máximo. 

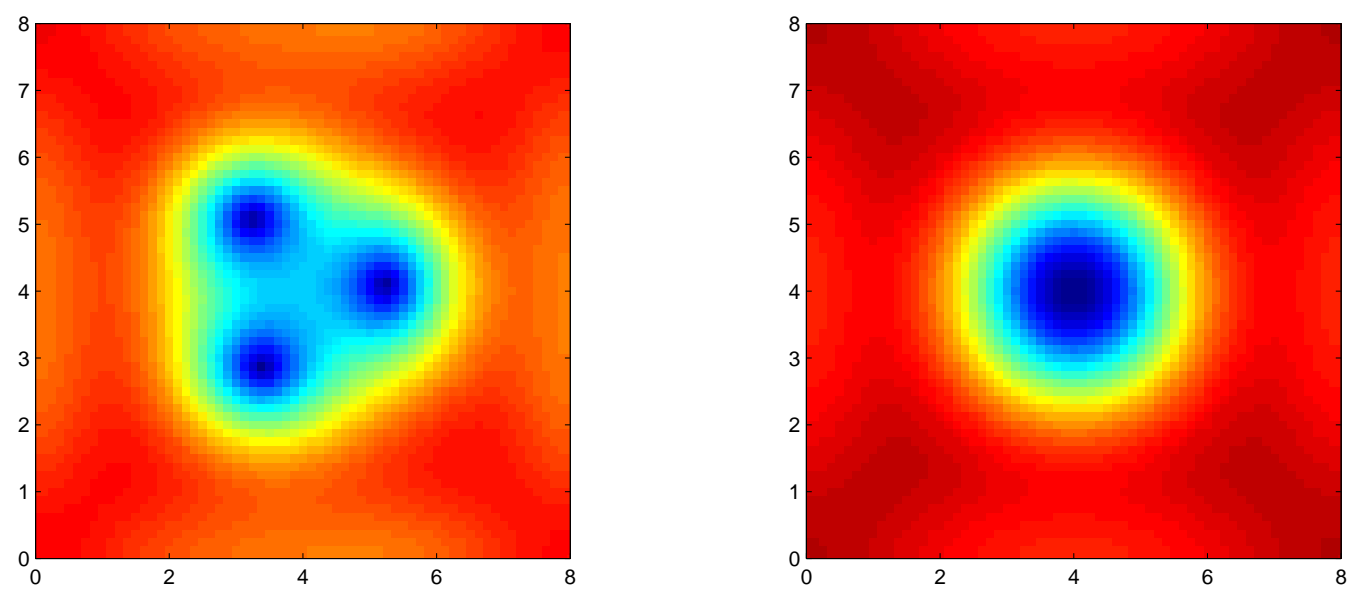

Figura 2.9: Topologia do parâmetro de ordem $|\psi|$ : estado de multivórtice (esquerda) e de vórtice gigante (direita) com vorticidade 3. Em ambas as figuras as dimensões são $8 \xi \times 8 \xi$.

Para amostras suficientemente pequenas, os vórtices podem se sobrepor tão fortemente que é mais favorável energeticamente formar apenas um vórtice com múltipla vorticidade. Esta situação é conhecida como vórtice gigante, o qual contém mais de um quantum de fluxo. A Figura 2.9 mostra um vórtice gigante no centro da amostra. A existência de estados de vórtices gigantes ainda é um assunto controverso e que necessita de mais investigações. No Capítulo 4 exploraremos este problema em mais detalhes para o caso de filmes finos de superfícies variáveis. 


\section{Capítulo 3}

\section{Método de Variáveis de Ligação para as Equações TDGL}

\subsection{Introdução}

Com o advento de computadores digitais com alta velocidade de processamento, nas últimas décadas a simulação computacional tem sido muito utilizada para fazer previsões de sistemas físicos reais, em particular para o estudo do fenômeno da supercondutividade. Muito embora simulações não reflitam o comportamento real de um supercondutor, partindo de uma situação ideal, podemos ir incrementando à simulação detalhes que se assemelham à situação real e chegar a bons resultados. Apesar das limitações computacionais, a janela de possibilidades que podem ser simuladas são inúmeras. Em seguida, listamos algumas amostras supercondutoras que vêm sendo exploradas por muitos autores:

- amostras com diferentes formas geométricas;

- amostras com alguns defeitos (buracos) distribuídos de forma regular ou aleatória;

- amostras com impurezas magnéticas distribuídas de forma regular. 
Método de Variáveis de Ligação ... 43

Vale a pena lembrar também que, atualmente, a confecção de amostras supercondutoras com dimensões bastante pequenas já é uma realidade, o que torna possível a comparação entre os dados reais com os simulados.

Um método muito conhecido e utilizado, são as soluções numéricas das equações de Ginzburg-Landau que descrevem de uma forma bastante satisfatória o comportamento supercondutor de amostras metálicas ou mesmo os supercondutores de alta temperatura crítica. Nas próximas Seções iremos desenvolver um método que permite solucionar estas equações numericamente.

\subsection{As Equações TDGL}

Para o estudo da supercondutividade utilizamos as equações de Ginzburg-Landau dependentes do tempo (TDGL). Estas equações são a generalização das equações (2.29), (2.31) e (2.32) para a supercondutividade de não-equilíbrio [22, 23]. Em 1966, Schmid [24] propôs a seguinte extensão das equações de Ginzburg-Landau:

$$
\begin{gathered}
\frac{\hbar}{2 m^{*} D}\left(\frac{\partial}{\partial t}+i \frac{e^{*}}{\hbar} \Phi\right) \psi=-\frac{1}{2 m^{*}}\left(-i \hbar \boldsymbol{\nabla}-\frac{e^{*}}{c} \mathbf{A}\right)^{2} \psi+\alpha \psi+\beta|\psi|^{2} \psi \\
\frac{4 \pi \sigma}{c}\left(\frac{1}{c} \frac{\partial \mathbf{A}}{\partial t}+\nabla \Phi\right)=\frac{4 \pi}{c} \mathbf{J}_{s}-\nabla \times \nabla \times \mathbf{A}
\end{gathered}
$$

onde $\mathrm{D}$ é conhecido como coeficiente de difusão, $\sigma$ é a condutividade elétrica, $\Phi$ é o potencial escalar e $\mathbf{J}_{s}$ é a densidade de corrente supercondutora dada por:

$$
\mathbf{J}_{s}=\frac{e^{*}}{m^{*}} \operatorname{Re}\left[\bar{\psi}\left(\frac{\hbar}{i} \boldsymbol{\nabla}-\frac{e^{*}}{c} \mathbf{A}\right) \psi\right],
$$

onde Re denota a parte real de uma quantidade complexa.

Note que a equação (3.2) também pode ser escrita na forma da lei de Ampère:

$$
\nabla \times \mathbf{B}=\frac{4 \pi}{c} \mathbf{J}
$$


onde $\mathbf{J}=\sigma \mathbf{E}+\mathbf{J}_{s}, \mathbf{E}=-(1 / c) \partial \mathbf{A} / \partial t-\boldsymbol{\nabla} \Phi$ é o campo elétrico, ou seja, a corrente total $\mathbf{J}$ pode ser interpretada como a soma de uma corrente normal a qual satisfaz a lei de Ohm, $\mathbf{J}_{N}=\sigma \mathbf{E}$, e uma supercorrente $\mathbf{J}_{s}$.

Do ponto de vista computacional, é conveniente trabalhar com equações que apresentam variáveis na forma adimensional. Isto pode ser feito fazendo as seguintes mudanças de variáveis:

$$
\begin{aligned}
\psi & =\psi_{0} \tilde{\psi} \\
T & =T_{c} \tilde{T} \\
\boldsymbol{\nabla} & =\frac{1}{\xi(0)} \tilde{\boldsymbol{\nabla}}, \\
\mathbf{A} & =H_{c 2}(0) \xi(0) \tilde{\mathbf{A}} \\
\Phi & =\frac{H_{c 2}(0) D}{c} \tilde{\Phi},
\end{aligned}
$$

onde $\psi_{0}^{2}=\alpha / \beta=(a / \beta) T_{c}\left(1-T / T_{c}\right)$.

Assim, as equações TDGL assumem a forma:

$$
\begin{gathered}
\frac{\partial \psi}{\partial t}+i \Phi \psi=-(-i \nabla-\mathbf{A})^{2} \psi+(1-T) \psi\left(1-|\psi|^{2}\right), \\
\beta\left(\frac{\partial \mathbf{A}}{\partial t}+\nabla \Phi\right)=(1-T) \operatorname{Re}[\bar{\psi}(-i \boldsymbol{\nabla}-\mathbf{A}) \psi]-\kappa^{2} \boldsymbol{\nabla} \times \boldsymbol{\nabla} \times \mathbf{A}
\end{gathered}
$$

onde, depois de feitas as substituições (3.5), suprimimos os til's das novas equações; a barra indica o complexo conjugado da respectiva função complexa; o parâmetro $\beta=4 \pi \sigma D \lambda^{2}(0) / c^{2} \xi^{2}(0)$.

Note que as equações (3.6) e (3.7) são invariantes sob as transformações de calibre $\psi^{\prime}=\psi e^{i \chi}, \mathbf{A}^{\prime}=\mathbf{A}+\nabla \chi, \Phi^{\prime}=\Phi-\partial \chi / \partial t$, onde $\chi$ é uma função arbitrária. Escolheremos o calibre onde $\Phi^{\prime}=0$ para todo instante de tempo $t$. Abandonando as linhas, obtemos as equações:

$$
\frac{\partial \psi}{\partial t}=-(-i \nabla-\mathbf{A})^{2} \psi+(1-T) \psi\left(1-|\psi|^{2}\right)
$$


Método de Variáveis de Ligação ...

$$
\begin{gathered}
\beta \frac{\partial \mathbf{A}}{\partial t}=\mathbf{J}_{s}-\kappa^{2} \boldsymbol{\nabla} \times \boldsymbol{\nabla} \times \mathbf{A}, \\
\mathbf{J}_{s}=(1-T) \operatorname{Re}[\bar{\psi}(-i \boldsymbol{\nabla}-\mathbf{A}) \psi] .
\end{gathered}
$$

Neste ponto, é conveniente a introdução dos campos auxiliares, que são definidos por: ${ }^{1}$

$$
\begin{aligned}
& \mathcal{U}^{x}(x, y, t)=\exp \left(-i \int_{x_{0}}^{x} A_{x}(\xi, y, t) d \xi\right) \\
& \mathcal{U}^{y}(x, y, t)=\exp \left(-i \int_{y_{0}}^{y} A_{y}(x, \eta, t) d \eta\right)
\end{aligned}
$$

onde $\left(x_{0}, y_{0}\right)$ é um ponto arbitrário.

Em termos dos campos auxiliares, [25, 26] a equação (3.8) pode ser reescrita como:

$$
\frac{\partial \psi}{\partial t}=\overline{\mathcal{U}}_{x} \frac{\partial^{2}}{\partial x^{2}}\left(\mathcal{U}_{x} \psi\right)+\overline{\mathcal{U}}_{y} \frac{\partial^{2}}{\partial y^{2}}\left(\mathcal{U}_{y} \psi\right)+(1-T) \psi\left(1-|\psi|^{2}\right)
$$

A segunda equação (3.9) permanece inalterada, exceto pelo fato que agora a densidade de corrente supercondutora toma a forma:

$$
J_{s \mu}=(1-T) \operatorname{Im}\left[\overline{\mathcal{U}}_{\mu} \bar{\psi} \frac{\partial}{\partial \mu}\left(\mathcal{U}_{\mu} \psi\right)\right]
$$

onde $\mu=x, y$ e Im indica a parte imaginária.

\subsubsection{Método das Variáveis de Ligação}

Uma vez que as equações TDGL não apresentam em geral soluções analíticas, é importante o uso de métodos numéricos. [25-28] Neste contexto, um método muito conhecido é o das diferenças finitas. Este método consiste em utilizar uma malha geralmente retangular $N_{x} \times N_{y}$ com células de dimensões $a_{x} \times a_{y}$; este método também pode ser empregado para malhas com outros formatos. Quanto menor for os valores de $a_{x}$ e $a_{y}$ melhor será o resultado; em outras palavras, mais nos aproximamos

\footnotetext{
${ }^{1}$ A partir deste ponto, restringiremos nosso desenvolvimento das TDGL's em duas dimensões, embora a generalização para o caso tridimensional seja trivial.
} 
da solução exata. As equações TDGL são resolvidas levando em consideração as condições iniciais para o parâmetro de ordem $\psi$ e o potencial vetor $\mathbf{A}$, e as condições de contorno que dependem de cada problema a ser estudado.

Para resolver as equações TDGL é necessário substituir variáveis contínuas por discretas equivalentes. Para garantir a consistência do método, é necessário definir em quais pontos da malha quais quantidades físicas serão calculadas. Como as equações TDGL são equações diferenciais que apresentam invariância de calibre, é necessário um certo cuidado, pois ao discretizarmos tais equações, pode ocorrer quebra dessa invariância (quebra de simetria) e os resultados poderão não ser satisfatórios (não físicos). O uso das chamadas variáveis de ligação que apresentaremos mais adiante garantem a invariância de calibre no processo de discretização. [28]

Antes de prosseguirmos com a discretização, será importante definirmos as variáveis de ligação. Considere dois pontos adjacentes $\left(x_{i}, y_{j}\right)$ e $\left(x_{i+1}, y_{j}\right)$ da malha de discretização:

$$
\begin{aligned}
U_{x, i, j} & =\mathcal{U}_{x}\left(x_{i+1}, y_{j}\right) \overline{\mathcal{U}}_{x}\left(x_{i}, y_{j}\right) \\
& =\exp \left(-i \int_{x_{i}}^{x_{i+1}} A_{x}\left(\xi, y_{j}\right) d \xi\right)
\end{aligned}
$$

onde omitimos a dependência temporal.

Analogamente, ao longo da vertical temos a definição:

$$
\begin{aligned}
U_{y, i, j} & =\mathcal{U}_{y}\left(x_{i}, y_{j+1}\right) \overline{\mathcal{U}}_{y}\left(x_{i}, y_{j}\right) \\
& =\exp \left(-i \int_{y_{j}}^{y_{j+1}} A_{y}\left(x_{i}, \eta\right) d \eta\right) .
\end{aligned}
$$

Usando a regra do ponto médio para integração simples, também podemos escrever:

$$
\begin{aligned}
& U_{x, i, j}=\exp \left(-i A_{x, i, j} a_{x}\right), \\
& U_{y, i, j}=\exp \left(-i A_{y, i, j} a_{y}\right),
\end{aligned}
$$


onde $A_{x, i, j}=A_{x}\left(x_{i}+a_{x} / 2, y_{j}\right)$ e $A_{y, i, j}=A_{y}\left(x_{i}, y_{j}+a_{y} / 2\right)$.

Considere uma grade retangular de $N_{x} \times N_{y}$ células unitárias com dimensões $a_{x} \times a_{y}$ como ilustra a Figura 3.1. A região supercondutora da amostra é delimitada pelo tracejado dentro da malha. A região delimitada pelas linhas tracejada e sólida mais externa é uma fina camada "metálica", e o exterior é considerado como vácuo. ${ }^{2}$ Os pontos onde cada quantidade física é calculada estão devidamente indicados na Figura 3.1. Nos vértices das células unitárias, calculamos o parâmetro de ordem $\psi$. Nos pontos médios ao longo da horizontal entre um vértice e outro calculamos as variáveis de ligação $U_{x}$. Similarmente, ao longo da vertical calculamos as variáveis de ligação $U_{y}$.

O cálculo da integral de caminho do potencial vetor numa célula unitária da malha tem como resultado o fluxo magnético. De fato, considere um domínio $\mathscr{D}=$ $\left\{x_{i}<x<x_{i+1}, y_{j}<y<y_{j+1}\right\}$, exatamente uma célula unitária. Seja $\partial \mathscr{D}$ os contornos de $\mathscr{D}$. Então temos que:

$$
\begin{aligned}
\exp \left(-i \int B_{z} d x d y\right) & =\exp \left(-i \oint_{\partial \mathscr{D}} \mathbf{A} \cdot d \mathbf{r}\right) \\
& =U_{x, i, j} U_{y, i+1, j} \bar{U}_{x, i, j+1} \bar{U}_{y, i, j} \\
& \equiv L_{i, j} .
\end{aligned}
$$

Por outro lado, usando a regra do ponto médio para integração dupla, temos que:

$$
\begin{aligned}
\exp \left(-i \int B_{z} d x d y\right) & =\exp \left(-i B_{z, i, j} a_{x} a_{y}\right) \\
& =1-i B_{z, i, j} a_{x} a_{y}
\end{aligned}
$$

\footnotetext{
${ }^{2}$ De fato, toda a amostra é considerada do mesmo material supercondutor. Esta separação da fina camada metálica é um artifício matemático para evitar de aplicar as condições de contorno na interface supercondutor-vácuo, pois alí a derivada do parâmetro de ordem é infinita. Assim, os pontos externos da malha atuam como pontos falsos.
} 
onde $B_{z, i, j}=B_{z}\left(x_{i}+a_{x} / 2, y_{j}+a_{y} / 2\right)$. Desta última equação obtemos:

$$
B_{z, i, j}=-\frac{1}{a_{x} a_{y}} \operatorname{Im}\left(L_{i, j}\right)
$$

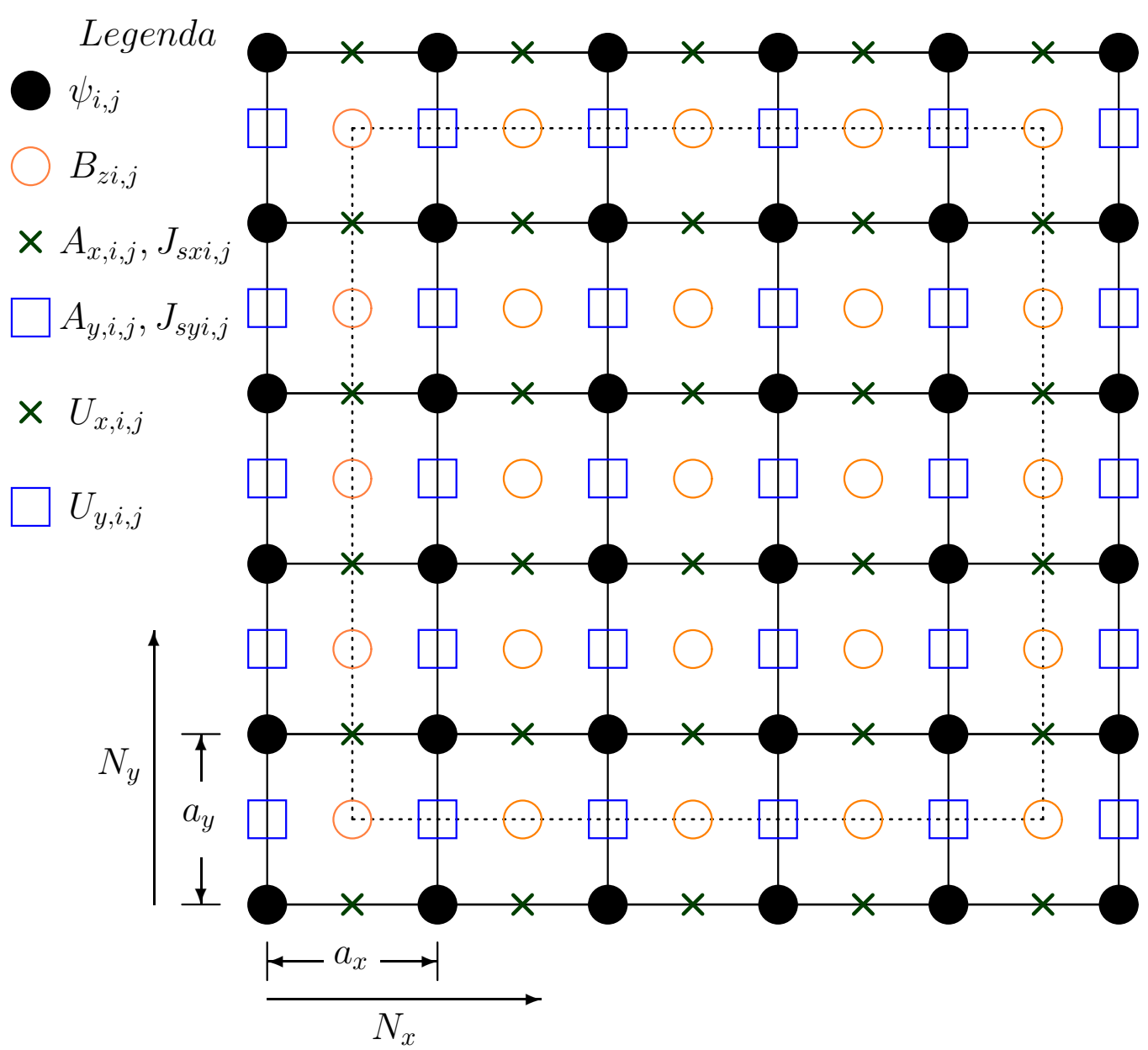

Figura 3.1: Malha de discretização.

No que segue abaixo, listamos as variáveis discretas que usaremos na discretização das equações TDGL, bem como indicamos os pontos onde elas serão calculadas:

- $\psi_{i, j}$, com $1 \leq i \leq N_{x}+1,1 \leq j \leq N_{y}+1$, associados aos nós ou vértices da malha. O valor de $\psi_{i, j}$ corresponde ao valor do parâmetro de ordem na posição $\left(x_{i}, y_{j}\right)$ 
- $U_{i, j}^{x}$ (variável de ligação na direção $x$, com $1 \leq i \leq N_{x}, 1 \leq j \leq N_{y}+1$, associado às linhas horizontais);

- $U_{i, j}^{y}$ (variável de ligação na direção $y$, com $1 \leq i \leq N_{x}+1,1 \leq j \leq N_{y}$, associado às linhas verticais).

- $A_{x, i, j}$ e $J_{s x, i, j}$, com $1 \leq i \leq N_{x}, 1 \leq j \leq N_{y}+1$, associados aos pontos médios entre dois vértices consecutivos ao longo das linhas horizontais;

- $A_{y, i, j}$ e $J_{s y, i, j}$, com $1 \leq i \leq N_{x}+1,1 \leq j \leq N_{y}$, associados aos pontos médios entre dois vértices consecutivos ao longo das linhas verticais;

- $B_{z, i, j}$, com $1 \leq i \leq N_{x}, 1 \leq j \leq N_{y}$, associados aos centros das células unitárias.

Estas considerações iniciais serão suficientes para iniciarmos o processo de discretização das equações. Comecemos pela equação (3.13). Usando a aproximação de segunda ordem para a primeira derivada: [29]

$$
f^{\prime}(x)=\frac{f(x+\Delta x / 2)-f(x-\Delta x / 2)}{\Delta x}
$$

após algumas manipulações algébricas, obtemos:

$$
\begin{aligned}
\overline{\mathcal{U}}_{x} \frac{\partial^{2}}{\partial x^{2}}\left(\mathcal{U}_{x} \psi\right)+\left.\overline{\mathcal{U}}_{y} \frac{\partial^{2}}{\partial y^{2}}\left(\mathcal{U}_{y} \psi\right)\right|_{\left(x_{i}, y_{j}\right)}= & \frac{U_{x, i, j} \psi_{i+1, j}-2 \psi_{i, j}+\bar{U}_{x, i-1, j} \psi_{i-1, j}}{a_{x}^{2}} \\
& +\frac{U_{y, i, j+1} \psi_{i, j+1}-2 \psi_{i, j}+\bar{U}_{y, i, j-1} \psi_{i, j-1}}{a_{y}^{2}}
\end{aligned}
$$

Assim, a primeira equação TDGL (3.13) calculada no ponto $\left(x_{i}, y_{j}\right)$ toma a forma:

$$
\begin{aligned}
\frac{\partial \psi_{i, j}}{\partial t}= & \frac{U_{x, i, j} \psi_{i+1, j}-2 \psi_{i, j}+\bar{U}_{x, i-1, j} \psi_{i-1, j}}{a_{x}^{2}} \\
& +\frac{U_{y, i, j+1} \psi_{i, j+1}-2 \psi_{i, j}+\bar{U}_{y, i, j-1} \psi_{i, j-1}}{a_{y}^{2}} \\
& +(1-T) \psi_{i, j}\left(1-\left|\psi_{i, j}\right|^{2}\right)
\end{aligned}
$$


Agora, passamos a discretizar a equação (3.9). Usando a aproximação para a derivada (3.21) e a equação da densidade de corrente (3.14), encontramos:

$$
\begin{aligned}
J_{s x, i, j} & =J_{s x}\left(x_{i}+a_{x} / 2, y_{j}\right) \\
& =\left.(1-T) \operatorname{Im}\left[\overline{\mathcal{U}}_{x} \bar{\psi} \frac{\partial}{\partial x}\left(\mathcal{U}_{x} \psi\right)\right]\right|_{\left(x_{i}+a_{x} / 2, y_{j}\right)} \\
& =\frac{1-T}{a_{x}} \operatorname{Im}\left[\bar{\psi}_{i, j} U_{x, i, j} \psi_{i+1, j}\right]
\end{aligned}
$$

$\mathrm{e}$

$$
\begin{aligned}
J_{s y, i, j} & =J_{s y}\left(x_{i}, y_{j}+a_{y} / 2\right) \\
& =\left.(1-T) \operatorname{Im}\left[\overline{\mathcal{U}}_{y} \bar{\psi} \frac{\partial}{\partial y}\left(\mathcal{U}_{y} \psi\right)\right]\right|_{\left(x_{i}, y_{j}+a_{y} / 2\right)} \\
& =\frac{1-T}{a_{y}} \operatorname{Im}\left[\bar{\psi}_{i, j} U_{y, i, j} \psi_{i, j+1}\right] .
\end{aligned}
$$

Lembrando que $(\boldsymbol{\nabla} \times \boldsymbol{\nabla} \times \mathbf{A})_{x}=(\boldsymbol{\nabla} \times \mathbf{B})_{x}=\partial B_{z} / \partial y$, e novamente usando a aproximação para a primeira derivada, temos que:

$$
\begin{aligned}
\left.\frac{\partial}{\partial y} B_{z}\right|_{\left(x_{i}+a_{x} / 2, y_{j}\right)} & =\frac{B_{z}\left(x_{i}+a_{x} / 2, y_{j}+a_{y} / 2\right)-B_{z}\left(x_{i}+a_{x} / 2, y_{j}-a_{y} / 2\right)}{a_{y}} \\
& =\frac{B_{z, i, j}-B_{z, i, j-1}}{a_{y}} .
\end{aligned}
$$

Analogamente, podemos determinar $(\boldsymbol{\nabla} \times \boldsymbol{\nabla} \times \mathbf{A})_{y}=(\boldsymbol{\nabla} \times \mathbf{B})_{y}=-\partial B_{z} / \partial x$. Temos que:

$$
\begin{aligned}
\left.\frac{\partial}{\partial x} B_{z}\right|_{\left(x_{i}, y_{j}+a_{y} / 2\right)} & =\frac{B_{z}\left(x_{i}+a_{x} / 2, y_{j}+a_{y} / 2\right)-B_{z}\left(x_{i}-a_{x} / 2, y_{j}-a_{y} / 2\right)}{a_{x}} \\
& =\frac{B_{z, i, j}-B_{z, i-1, j}}{a_{x}} .
\end{aligned}
$$

Finalmente, calculando a segunda equação TDGL, componente $x$ no ponto $\left(x_{i}+\right.$ $\left.a_{x} / 2, y_{j}\right)$ e componente $y$ no ponto $\left(x_{i}, y_{j}+a_{y} / 2\right)$, obtemos as equações discretizadas:

$$
\beta \frac{\partial}{\partial t} A_{x, i, j}=\frac{1-T}{a_{x}} \operatorname{Im}\left[\bar{\psi}_{i, j} U_{x, i, j} \psi_{i+1, j}\right]-\frac{\kappa^{2}}{a_{y}}\left[B_{z, i, j}-B_{z, i, j-1}\right]
$$


Método de Variáveis de Ligação ...

$\mathrm{e}$

$$
\beta \frac{\partial}{\partial t} A_{y, i, j}=\frac{1-T}{a_{y}} \operatorname{Im}\left[\bar{\psi}_{i, j} U_{y, i, j} \psi_{i, j+1}\right]+\frac{\kappa^{2}}{a_{x}}\left[B_{z, i, j}-B_{z, i-1, j}\right]
$$

Para finalizar a discretização, precisamos de criar relações de recorrência temporal entre as quantidades físicas. Suponhamos que conhecemos, por exemplo, o parâmetro de ordem $\psi_{i, j}$ em $t=t_{n}$ e queiramos determiná-lo em $t=t_{n+1}$. Denotemos por $\psi_{i, j}^{n}=\psi_{i, j}\left(t_{n}\right)$, e similarmente para as outras quantidades físicas. Usando a aproximação de primeira ordem: [29]

$$
\int_{t_{n}}^{t_{n+1}} f(t) d t=f_{n} \Delta t, \quad \Delta t=t_{n+1}-t_{n},
$$

da primeira equação TDGL discretizada no espaço (3.23) encontramos a relação de recorrência:

$$
\begin{aligned}
\psi_{i, j}^{n+1}= & \psi_{i, j}^{n}+\Delta t\left[\frac{U_{x, i, j}^{n} \psi_{i+1, j}^{n}-2 \psi_{i, j}^{n}+\bar{U}_{x, i-1, j}^{n} \psi_{i-1, j}^{n}}{a_{x}^{2}}\right. \\
& +\frac{U_{y, i, j+1}^{n} \psi_{i, j+1}^{n}-2 \psi_{i, j}^{n}+\bar{U}_{y, i, j-1}^{n} \psi_{i, j-1}^{n}}{a_{y}^{2}} \\
& \left.+(1-T) \psi_{i, j}\left(1-\left|\psi_{i, j}\right|^{2}\right)\right]
\end{aligned}
$$

Esta relação aplica-se para os pontos interiores da malha $2 \leq i \leq N_{x}, 2 \leq j \leq N_{y}$. Os pontos mais exteriores serão determinados pelas condições de contorno. De forma idêntica podemos determinar as relações de recorrência para as componentes do potencial vetor:

$$
A_{x, i, j}^{n+1}=A_{x, i, j}^{n}+\Delta t\left\{\frac{1-T}{a_{x}} \operatorname{Im}\left[\bar{\psi}_{i, j}^{n} U_{x, i, j}^{n} \psi_{i+1, j}^{n}\right]-\frac{\kappa^{2}}{a_{y}}\left[B_{z, i, j}^{n}-B_{z, i, j-1}^{n}\right]\right\},
$$

e

$$
A_{y, i, j}^{n+1}=A_{y, i, j}^{n}+\Delta t\left\{\frac{1-T}{a_{y}} \operatorname{Im}\left[\bar{\psi}_{i, j}^{n} U_{y, i, j}^{n} \psi_{i, j+1}^{n}\right]+\frac{\kappa^{2}}{a_{x}}\left[B_{z, i, j}^{n}-B_{z, i-1, j}^{n}\right]\right\}
$$

onde a primeira equação acima vale para todos os pontos $1 \leq i \leq N_{x}, 2 \leq j \leq N_{y}$ e a segunda para $2 \leq i \leq N_{x}, 1 \leq j \leq N_{y}$. 


\subsubsection{Condições de Contorno}

Devemos impor que não haja densidade de corrente perpendicular à superfície da amostra (linha tracejada da Figura 3.1). Isso implica em escrever que:

$$
\begin{aligned}
\left.\operatorname{Im}\left[\overline{\mathcal{U}}_{x} \bar{\psi} \frac{\partial}{\partial x}\left(\mathcal{U}_{x} \psi\right)\right]\right|_{\left(x_{1}+a_{x} / 2, y_{j}\right)} & =0, \text { lado oeste da malha } \\
\left.\operatorname{Im}\left[\overline{\mathcal{U}}_{x} \bar{\psi} \frac{\partial}{\partial x}\left(\mathcal{U}_{x} \psi\right)\right]\right|_{\left(x_{N_{x}}+a_{x} / 2, y_{j}\right)} & =0, \text { lado leste da malha } \\
\left.\operatorname{Im}\left[\overline{\mathcal{U}}_{y} \bar{\psi} \frac{\partial}{\partial y}\left(\mathcal{U}_{y} \psi\right)\right]\right|_{\left(x_{i}, y_{1}+a_{y} / 2\right)} & =0, \text { lado sul da malha }, \\
\left.\operatorname{Im}\left[\overline{\mathcal{U}}_{y} \bar{\psi} \frac{\partial}{\partial y}\left(\mathcal{U}_{y} \psi\right)\right]\right|_{\left(x_{i}, y_{N_{y}}+a_{y} / 2\right)} & =0, \text { lado norte da malha } .
\end{aligned}
$$

Da aproximação para a primeira derivada (3.21), uma simples álgebra nos conduz às seguintes relações:

$$
\begin{aligned}
\psi_{1, j}^{n+1} & =U_{x, 1, j}^{n+1} \psi_{2, j}^{n+1}, \text { lado oeste da malha }, \\
\psi_{N_{x}+1, j}^{n+1} & =\bar{U}_{x, N_{x}, j}^{n+1} \psi_{N_{x}, j}^{n+1}, \quad \text { lado leste da malha }, \\
\psi_{i, 1}^{n+1} & =U_{y, 1, i}^{n+1} \psi_{i, 2}^{n+1}, \quad \text { lado sul da malha }, \\
\psi_{i, N_{y}+1}^{n+1} & =\bar{U}_{y, i, N_{y}}^{n+1} \psi_{i, N_{y}}^{n+1}, \quad \text { lado norte da malha . }
\end{aligned}
$$

Estas relações aplicam-se a todos os pontos das bordas da malha, exceto os cantos. Para os vértices da amostra podemos aproximar o parâmetro de ordem, calculando as médias aritméticas dos dois parâmetros de ordem mais próximos. Entretanto, estes valores do parâmetro de ordem não são usados nas relações de recorrências. É importante também ressaltar que antes de usarmos estas condições de contorno, precisamos atualizar os valores das variáveis de ligação no interior da malha. Isto pode ser feito por meio das equações (3.16), uma vez que nestes pontos as componentes do potencial vetor já foram atualizadas através das relações de recorrência (3.32) e (3.33). 
O campo magnético pode ser atualizado nos pontos interiores da malha através das equações (3.18) e (3.20), pois nestes pontos as variáveis de ligação já foram calculadas em $t=t_{n+1}$. Nos pontos da fronteira (linha tracejada da Figura 3.1) simplesmente usamos $B_{z, i, j}^{n+1}=H$, onde $H$ é o campo externo aplicado.

Finalizando, devemos atualizar as variáveis de ligação nas bordas da malha. Para isto usamos a relação:

$$
U_{x, i, j} U_{y, i+1, j} \bar{U}_{x, i, j+1} \bar{U}_{y, i, j}=\exp \left(-i a_{x} a_{y} B_{z, i, j}\right)
$$

Em todas as células unitárias das bordas, com exceção das células dos cantos da malha, três das variáveis de ligação (3.36) são conhecidas, sendo que apenas uma delas requer correção que é a variável que se encontra na borda da malha. Isso é feito impondo que o valor do campo magnético deva ser igual ao campo aplicado de tal forma que mantemos a continuidade do campo.

Já nas células dos cantos da malha temos duas variáveis de ligação nas bordas da malha que requerem correção. Como neste caso temos duas incógnitas, optamos em escolher um dos valores que foram calculados no instante anterior.

As relações de recorrência não convergem para qualquer $\Delta t$. O critério para obter convergência é dado por: [25]

$$
\Delta t \leq \min \left\{\frac{\delta^{2}}{4}, \frac{\beta \delta^{2}}{4 \kappa^{2}}\right\},
$$

onde

$$
\delta^{2}=\frac{2}{\frac{1}{a_{x}^{2}}+\frac{1}{a_{y}^{2}}} .
$$

Notem que tão mais fina for a malha, mais iterações serão necessárias para obtermos o estado estacionário. Outro fator importante é o valor de $\kappa$. Para supercondutores fortemente do tipo-II, as simulações computacionais podem tornar-se inviáveis. 


\subsection{Filmes de Superfície Variável}

Foi demonstrado rigorosamente em alguns trabalhos matemáticos que para filmes supercondutores de espessura $2 \epsilon \leq 2 \xi(T)$, o campo magnético local é aproximadamente constante em todo o espaço de tal modo que as equações TDGL podem ser simplificadas significativamente. Denotemos o domínio de um filme supercondutor fino por $\Omega_{\epsilon}=\left\{(x, y, z) \in \mathscr{R}^{3}:(x, y) \in \Omega_{0}, \epsilon g_{1}(x, y)<z<\epsilon g_{2}(x, y)\right\}$, onde as funções $-1 \leq g_{1}(x, y) \leq 0,0 \leq g_{2}(x, y) \leq 1$ para todo $(x, y) \in \Omega_{0}$. Nas equações que se seguem denotaremos por $g(x, y)=\left[g_{2}(x, y)-g_{1}(x, y)\right] / 2$ como sendo a média entre entre as superfícies superior e inferior do filme. De acordo com Du [30-32] e seus colaboradores, ${ }^{3}$ até primeira ordem em $\epsilon$, as equações TDGL tornam-se:

$$
\begin{aligned}
\frac{\partial \psi}{\partial t}= & -\frac{1}{g}(-i \boldsymbol{\nabla}-\mathbf{A}) \cdot g(-i \boldsymbol{\nabla}-\mathbf{A}) \psi+(1-T) \psi\left(1-|\psi|^{2}\right), \text { em } \Omega \\
& \hat{\mathbf{n}} \cdot(-i \boldsymbol{\nabla}-\mathbf{A}) \psi=0, \text { sobre } \partial \Omega_{0},
\end{aligned}
$$

onde o campo magnético, até primeira ordem, é uniforme dentro do supercondutor, isto é, $\mathbf{B}=\mathbf{H}=\boldsymbol{\nabla} \times \mathbf{A}$. Conforme foi provado por Du e colaboradores, as equações (3.39) podem ser consideradas como boas aproximações para um filme supercondutor contanto que a condição $\epsilon \xi(T) / \lambda^{2}(T)=1\left(\epsilon \sqrt{1-T} / \kappa^{2}=1\right.$ em unidades reduzidas $)$ seja satisfeita. Pequenas correções para os campos dispersos fora do filme (stray fields) foram feitas pelos mesmos autores. Embora as correções para estes campos sejam importantes, elas não são determinantes para o comportamento de $\psi$. Consequentemente, para a determinação do parâmetro de ordem o campo magnético será considerado uniforme em todo o espaço. De fato, recentemente Brandt [17] demonstrou que no limite $\lambda^{2}(T) / 2 \epsilon a \geq 1$, onde $a$ é a metade da largura da amostra, a magnetização pode ser desconsiderada, isto é, a média do campo magnético dentro da amostra é aproximadamente igual ao campo externo $H$. Desta forma, não haverá

\footnotetext{
${ }^{3}$ Ver também Referência [33]
} 
necessidade de se utilizar a segunda equação de Ginzburg-Landau, o que nos leva a desconsiderar o valor do parâmetro $\kappa$. Na simulações computacionais apresentadas no próximo Capítulo, as escolhas dos parâmetros serão tais que satisfazem as desigualdades acima.

A primeira equação TDGL, mesmo a dependente da superfície do filme como em (3.39), deve também manter a invariância de calibre. Portanto, a reescreveremos em termos dos campos auxiliares. Temos que:

$$
\frac{\partial \psi}{\partial t}=\frac{1}{g}\left\{\bar{U}_{x} \frac{\partial}{\partial x}\left[g \frac{\partial}{\partial x}\left(U_{x} \psi\right)\right]+\bar{U}_{y} \frac{\partial}{\partial y}\left[g \frac{\partial}{\partial y}\left(U_{y} \psi\right)\right]\right\}+(1-T) \psi\left(1-|\psi|^{2}\right)
$$

ou ainda

$$
\begin{aligned}
\frac{\partial \psi}{\partial t}= & \frac{1}{g} \frac{\partial g}{\partial x} \bar{U}_{x} \frac{\partial}{\partial x}\left(U_{x} \psi\right)+\bar{U}_{x} \frac{\partial^{2}}{\partial x^{2}}\left(U_{x} \psi\right)+\frac{1}{g} \frac{\partial g}{\partial y} \bar{U}_{y} \frac{\partial}{\partial y}\left(U_{y} \psi\right)+\bar{U}_{y} \frac{\partial^{2}}{\partial y^{2}}\left(U_{y} \psi\right) \\
& +(1-T) \psi\left(1-|\psi|^{2}\right) .
\end{aligned}
$$

Os segundo e quarto termos do segundo membro da equação acima já foram discretizados anteriormente. Discretizando os demais termos e reescrevendo a equação acima, finalmente temos a forma discretizada para a primeira equação TDGL a qual leva em conta a variação da superfícies superior e inferior do filme. Assim, temos que:

$$
\begin{aligned}
\frac{\partial \psi_{i, j}}{\partial t}= & \frac{1}{g_{i, j}}\left(\frac{g_{i+1, j}-g_{i-1, j}}{2 a_{x}}\right)\left(\frac{U_{x, i, j} \psi_{i+1, j}-\bar{U}_{x, i-1, j} \psi_{i-1, j}}{2 a_{x}}\right) \\
& +\frac{U_{x, i, j} \psi_{i+1, j}-2 \psi_{i, j}+\bar{U}_{x, i-1, j} \psi_{i-1, j}}{a_{x}^{2}}+ \\
& \frac{1}{g_{i, j}}\left(\frac{g_{i, j+1}-g_{i, j-1}}{2 a_{y}}\right)\left(\frac{U_{y, i, j} \psi_{i, j+1}-\bar{U}_{y, i, j-1} \psi_{i, j-1}}{2 a_{y}}\right) \\
& +\frac{U_{x, i, j} \psi_{i+1, j}-2 \psi_{i, j}+\bar{U}_{x, i-1, j} \psi_{i-1, j}}{a_{x}^{2}} \\
& +(1-T) \psi_{i, j}\left(1-\left|\psi_{i, j}\right|^{2}\right) .
\end{aligned}
$$

As condições de contorno não se alteram com a introdução da variação das superfícies. 
No presente contexto, a magnetização foi calculada de acordo com a definição de momento magnético:

$$
\mathbf{M}=\frac{\mathbf{m}}{V}=\frac{1}{2 c} \int \mathbf{r} \times \mathbf{J}_{s} d^{3} r .
$$

Em termos da variáveis adimensionais, podemos escrever:

$$
\mathbf{M}=\frac{\mathbf{m}}{V}=\frac{1}{2} \frac{(1-T)}{4 \pi \kappa^{2} V} \int \mathbf{r} \times \mathbf{J}_{s} d^{3} r .
$$

No próximo Capítulo aplicaremos o presente algorítimo para vários casos de superfícies variáveis. 


\section{Capítulo 4}

\section{Estudo de Algumas Topologias de Filmes Finos Supercondutores}

\subsection{Introdução}

Importantes avanços nas técnicas de nanofabricação de supercondutores nas duas últimas décadas têm levado a um grande interesse na investigação de propriedades magnéticas de supercondutores de pequenas dimensões, da mesma ordem dos comprimentos característicos de penetração de London e de coerência.

A compreensão da movimentação de vórtices em supercondutores mesoscópicos é um trabalho extremamente complexo. A descrição de todo o processo, desde a primeira nucleação de um vórtice, até a acomodação de vários vórtices, depende de uma série de fatores. Podemos citar alguns deles: temperatura, campo magnético externo aplicado, tipo de material, geometria da amostra. Assim, o completo entendimento da dinâmica torna-se difícil. Além disso, a ancoragem (pinning) intrínseca devido ao processo de fabricação da amostra e rugosidade nas superfícies estão sempre presentes. Podemos também fabricar defeitos, na forma de uma rede de buracos (antidots). As vezes, buracos são muito importantes, já que eles aumentam a janela supercondutora. Em um supercondutor mesoscópico quadrado, eles podem produzir um campo crítico superior $H_{c 3}(T)$, três vezes maior do que o campo crítico 
superior $H_{c 2}(T)$ para um supercondutor volumétrico. [34]

Algumas configurações de vórtices que aparecem em supercondutores mesoscópicos são muitos diferentes da rede de Abrikosov. Em geral, a geometria da amostra influencia fortemente no arranjo de vórtices. Embora já fosse conhecido há muito tempo atrás que um vórtice gigante (vórtice com vorticidade superior a um) pudesse existir, em nível mesoscópico, foi simulado por Baelus e Peeters [18] que, em supercondutores livres de defeitos, esse estado realmente aparece. Nesta dissertação estudaremos as propriedades de um filme fino supercondutor mesoscópico quadrado de superfícies variáveis. Além da formação de vórtices gigantes, exploraremos a relação destes estados com a deformação das superfícies do filme. Também estudaremos o comportamento dos campos críticos com relação à variação dos parâmetros geométricos que controlam a forma das superfícies.

\subsection{Descrição das Topologias}

Tratamos aqui um filme como um paralelepípedo, cuja altura é muito menor do que as demais dimensões e naturalmente a altura será a espessura do filme. Com o objetivo de simular amostras tão reais quanto possível, assumiremos que a base (superfície inferior) do filme é sempre plana, tentando imitar o substrato num processo de fabricação de filmes finos por deposição. Entretanto, a face superior deixará de ser plana e assumirá três formas geométricas distintas. A primeira será um parabolóide com a concavidade voltada para baixo; a segunda, um parabolóide com a concavidade voltada para cima e, por último, uma superfície irregular (ou rugosa) gerada aleatoriamente.

As geometrias que investigaremos estão ilustradas na Figura 4.1. Observando a Figura 4.1a, vemos uma combinação de uma superfície côncava e um plano, e portanto denominaremos esta amostra de topologia plano-côncava. Para as Figuras 
4.1b e 4.1c, seguindo o mesmo raciocínio chamaremos de topologia plano-convexa e plano-irregular, respectivamente.

Cada uma destas topologias pode isoladamente ser convenientemente deformada por intermédio de parâmetros que chamaremos de $\alpha, \beta$ e $\gamma$ para cada geometria, respectivamente. Vamos denominá-los de parâmetros geométricos. As Figuras (4.2) demonstram estas propostas de forma que o parâmetro $\alpha$ varia a concavidade, o parâmetro $\beta$ varia a convexidade e o parâmetro $\gamma$ controla a porcentagem da rugosidade da superfície irregular. Na maioria dos casos, usaremos uma amostra quadrada de lados com dimensões fixas e iguais a 8 comprimentos de coerência $\xi$.

\subsection{Detalhes da Simulação}

A simulações computacionais foram realizadas segundo os passos especificados abaixo:

- A amostra de simulação é formada por uma malha quadrada de tamanho $8 \xi \times 8 \xi$, com células unitárias de tamanho $0.125 \xi \times 0.125 \xi$. Assim, temos uma malha com $64 \times 64$ células unitárias.

- Para determinarmos o comportamento temporal do campo magnético e do parâmetro de ordem necessitamos especificar qual o estado inicial do supercondutor. A condição inicial será impor que a amostra está no estado Meissner, e portanto o campo magnético $\mathbf{B}$ deve ser nulo em toda a amostra e o parâmetro de ordem $\psi$ deve valer 1 .

- O campo magnético aplicado $H$ é variado em passos pequenos desde 0 até atingir um valor em que a supercondutividade é completamente destruída, ou seja, $\psi \approx 0$, em todo o espaço, dentro de determinada precisão preestabelecida.

- Para cada valor de campo magnético o sistema é relaxado até encontar um estado estacionário, o qual pode corresponder a um estado estacionário ter- 

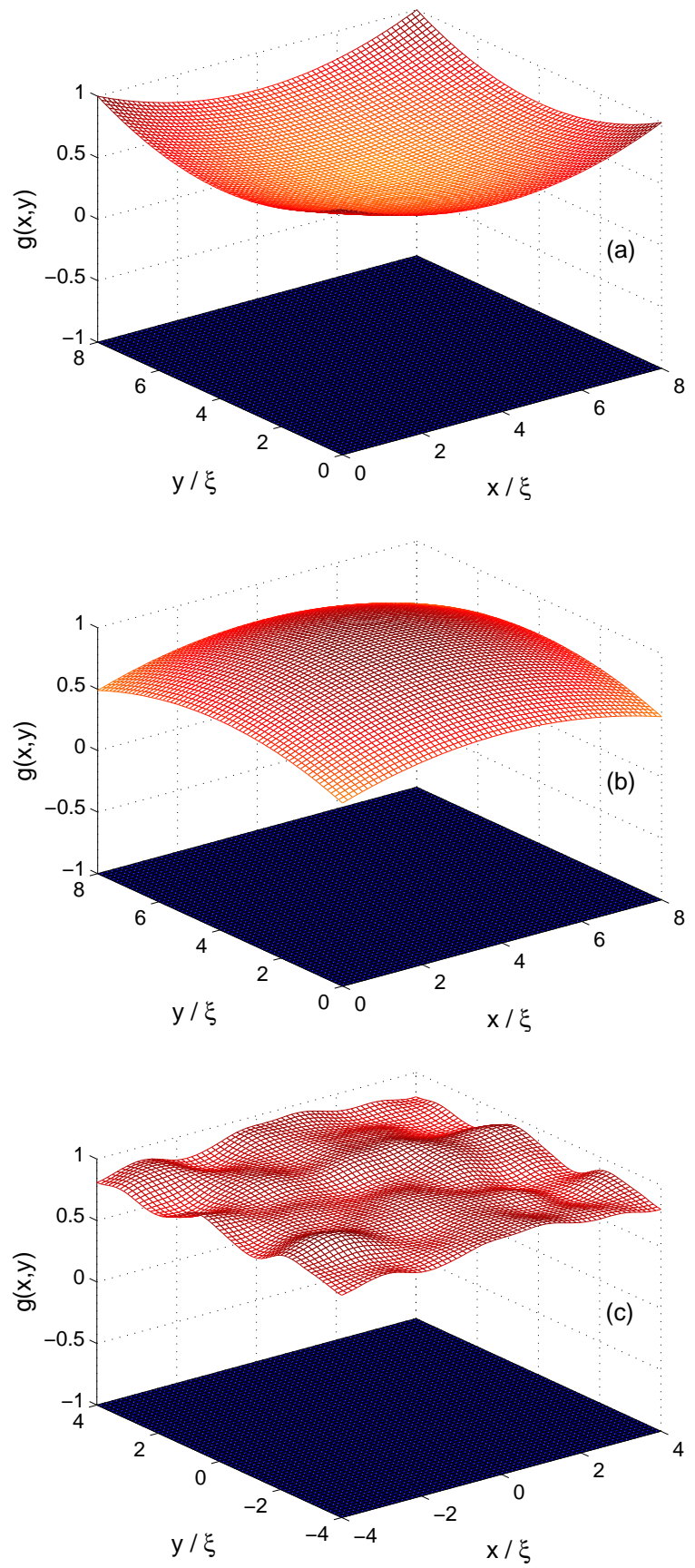

Figura 4.1: As três topologias usadas nas simulações: (a) plano-côncava, (b) planoconvexa, (c) plano-irregular. 


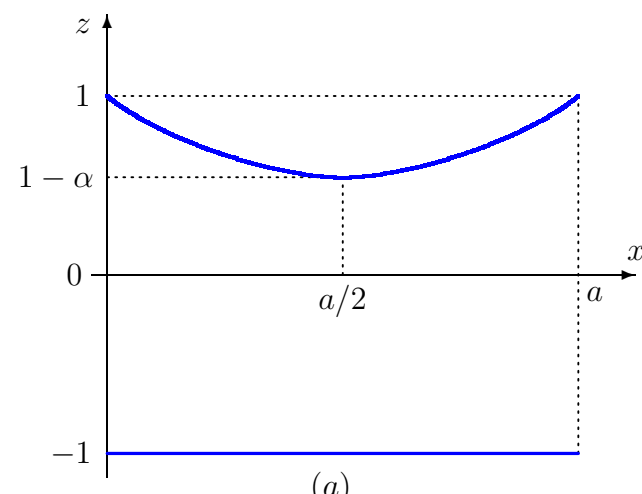

(a)

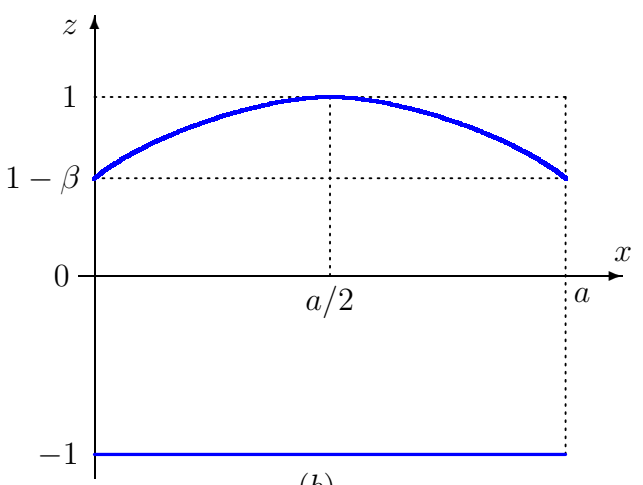

(b)

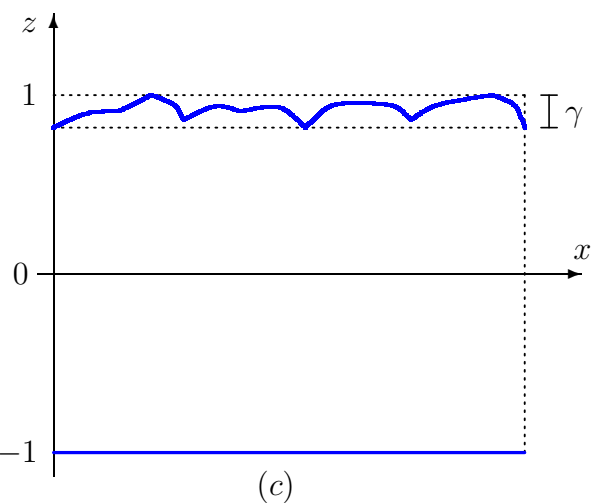

Figura 4.2: Visão frontal as três topologias (plano $y=b / 2$ ): (a) plano-côncava, (b) plano-Convexa, (c) plano-irregular.

modinâmico ou a um estado metaestável. O processo de relaxação é avaliado a partir das mudanças do parâmetro de ordem em todos os pontos da malha. A cada 1000 passos de integração, calculamos a variação do parâmetro de ordem em todos os pontos da malha. Se a maior variação for menor do que um valor $\epsilon$ preestabelecido, considera-se que o sistema entrou num estado de equilíbrio e a simulação prossegue, porém mudando para um novo valor de campo. Nas simulações aqui apresentadas, adotamos como critério $\epsilon=10^{-6}$.

- Para a topologia plano-côncava, usamos os seguintes valores para o parâmetro geométrico $\alpha=0.1,0.2,0.3, \ldots, 1.0$. Para a topologia plano-convexa, empregamos estes mesmos valores para o parâmetro geométrico $\beta$.

Finalmente, para a topologia plano-irregular, o parâmetro $\gamma$ assume os valores 
$1 \%, 5 \%, 10 \%, 15 \%, 20 \%$.

- Todas as simulações foram realizadas para a temperatura $T=0$. Assim, os resultados que apresentaremos são equivalentes a uma temperatura arbitrária caso tivessemos usado as equações TDGL com os comprimentos em função de $\xi(T){ }^{1}$

\subsection{Resultados e Discussões}

Para cada simulação, o programa implementado gerou vários arquivos trazendo para cada valor de campo magnético aplicado, os respectivos valores do parâmetro de ordem, da fase, da densidade de corrente e do número de vórtices. A análise destes arquivos permite acompanhar a evolução temporal das quantidades físicas relevantes desde o estado inicial para cada campo aplicado até o estado estacionário, passando pelos estados transientes.

\subsubsection{Comportamento dos Campos Críticos Inferior e Supe- rior}

De posse dos dados citados anteriormente, construímos dois gráficos dos campos críticos como função do respectivo parâmetro geométrico. As Figuras 4.3, 4.4 e 4.5, mostram o comportamento de $H_{c 1}$ e $H_{c 3}$ em função de $\alpha, \beta$ e $\gamma$, respectivamente.

Para a topologia plano-côncava, por intermédio do Figura 4.3a, podemos observar que, com o aumento de $\alpha$ o campo crítico inferior diminui, o que significa que a barreira para a primeira penetração é facilitada. Por outro lado, $H_{c 3}$ aumenta com o aumento de $\alpha$ (ver Figura 4.3b). Isso é muito importante do ponto de vista experimental, uma vez que o aumento nos campos críticos, leva a um aumento das

\footnotetext{
${ }^{1}$ Neste Capítulo, por simplicidade iremos usar as abreviações $\xi=\xi(0), H_{c 1}=H_{c 1}(0), H_{c 2}=$ $H_{c 2}(0)$ e $H_{c 3}=H_{c 3}(0)$.
} 

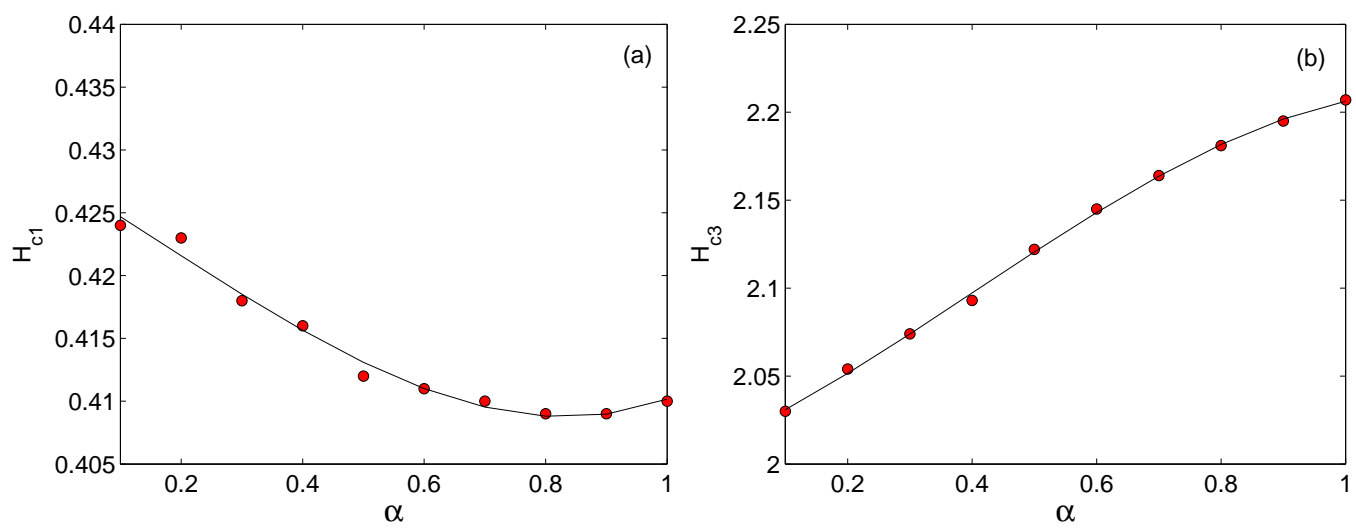

Figura 4.3: Campos críticos inferior (a) e superior (b) para a topologia planocôncava. A linha sólida representa uma interpolação polinomial

correntes críticas. A diminuição de $H_{c 1}$ não é significante, valendo apenas $3.3 \%$. Contudo, $H_{c 3}$ aumenta de $8.7 \%$.

As Figuras 4.4 exibem os campos críticos para a topologia plano-convexa. Podemos observar que para este caso ocorre exatamente o oposto com relação à topologia plano-côncava. O campo crítico inferior é aumentado de uma quantidade de $8.8 \%$ e o campo crítico superior diminui de 8.8\%. Em aplicações práticas, estas topologias mostraram ser interessantes, quando houver a necessidade de blindar o campo magnético aplicado.
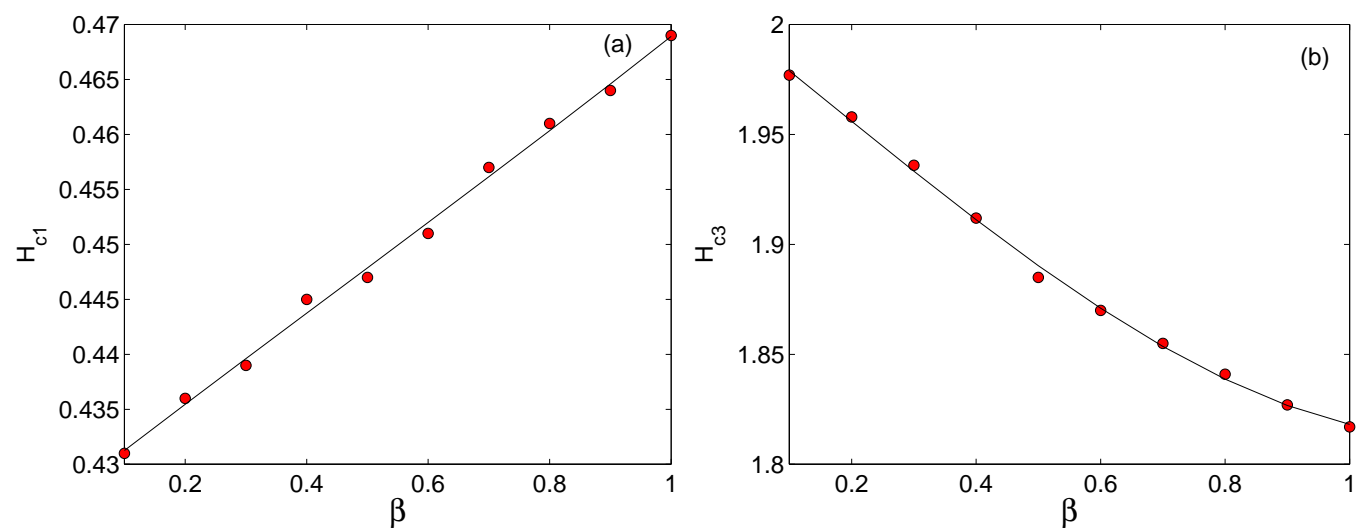

Figura 4.4: Campos críticos inferior (a) e superior (b) para a topologia planoconvexa. A linha sólida representa uma interpolação polinomial

Finalmente, para a topologia plano-irregular, podemos notar facilmente que os 
campos críticos não exibiram uma mudança substancial com relação aos filmes de superfícies planas. Entretanto, a curva de magnetização para esta geometria é a que mais se diferencia das outras como veremos na próxima Seção.
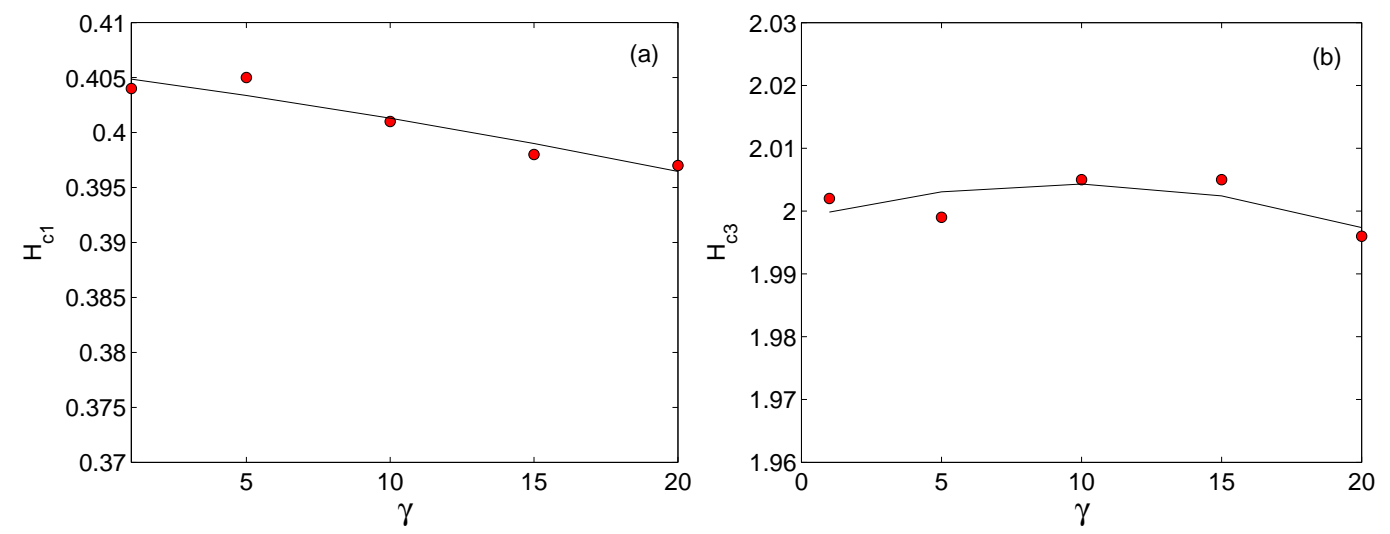

Figura 4.5: Campos críticos inferior (a) e superior (b) para a toplogia plano-irregular. A linha sólida representa uma interpolação polinomial

\subsubsection{Comportamento da Magnetização}

As figuras 4.6 apresentam as curvas de magnetização ${ }^{2}$ para as topologias descritas anteriormente. Cada salto na magnetização significa uma nucleação de um ou mais vórtices. Como podemos notar, as amostras plano-convexa com $\beta=1$ e aproximadamente plano-plano com $\beta=0.1$ apresentam transições bem distintas. Porém, para as outras topologias, seja qual for a perturbação da amostra, a magnetização tem aproximadamente o mesmo comportamento.

Da Figura 4.7, observamos que o comportamento da magnetização entre as distintas topologias é bem diferente. Isso evidencia que a forma geométrica tem uma forte influência no comportamento do supercondutor (configuração de vórtices, perfis de campo, etc.).

Denotaremos a vorticidade por $L$ e o campo de transição associado a cada salto

\footnotetext{
${ }^{2}$ Todas as curvas de magnetização estão normalizadas pelo seu respectivo máximo, pois tinhamos em mente visualizarmos apenas as transições supercondutoras.
} 

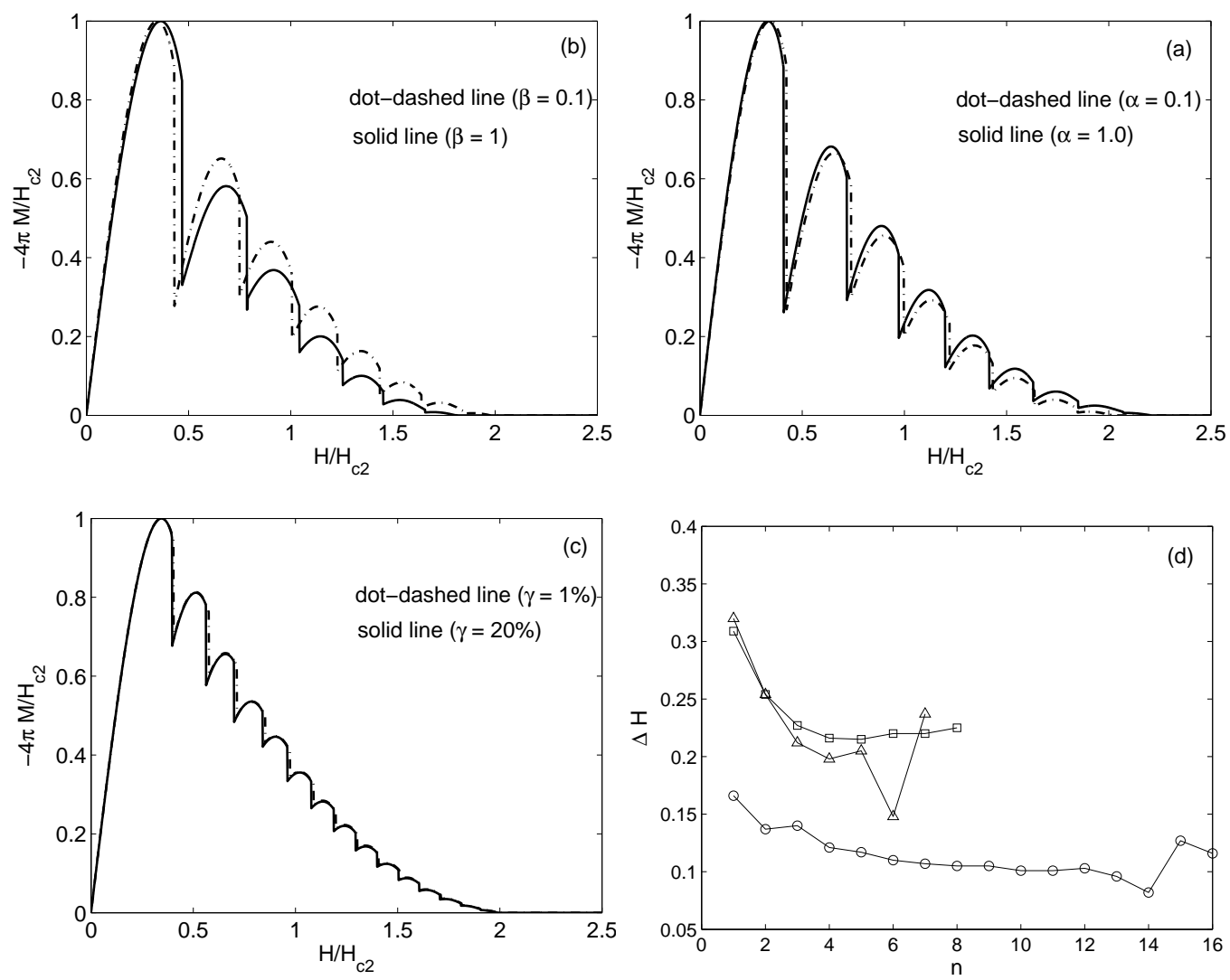

Figura 4.6: (a), (b) and (c) Magnetização em função do campo externo aplicado para os parâmetros geométricos conforme indicado nos painéis; (d) diferença de campo magnético $\Delta H$ em função da ordem de entrada de vórtices $n$, onde $(\square)$ plano-côncava , $(\triangle)$ plano-convexa, (o) plano-irregular.

nas curvas de magnetização por $H_{n}$. Graficamos $\Delta H=H_{n+1}-H_{n}$ (diferença de campos magnéticos entre uma transição e a subsequente) com função de $n$ (ver Figura 4.6d). Fica quase que evidente que para a topologia plano-irregular, $\Delta H$ é aproximadamente constante, indicando que a periodicidade nas transições é aproximadamente constante. Isto não é válido para as outras topologias.

Calculando os desvios padrões de $\Delta H$, para as três topologias, percebemos por meio do valor encontrado, que é menor para a plano-irregular, confirmando mais uma vez que a periodicidade é constante. De fato, para a as topologias plano-irregular, plano-côncava e plano-convexa os devios padrões da média são respectivamente, 


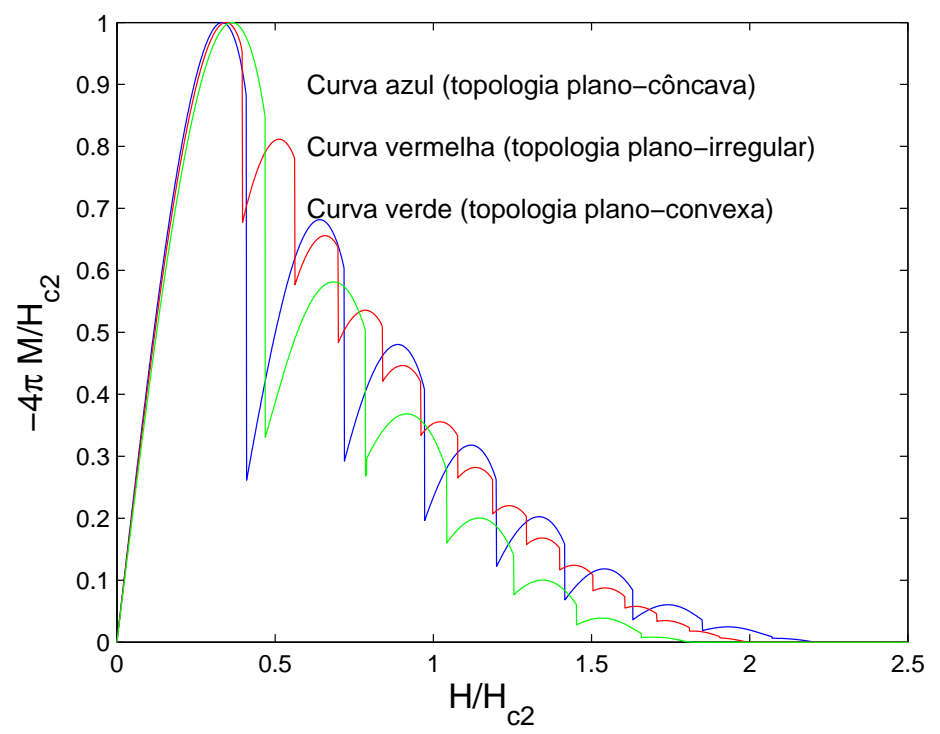

Figura 4.7: Magnetização em função do campo magnético aplicado para todas topologias na situação de maior deformação, $\alpha=\beta=1$ e $\gamma=20 \%$.

$0.020,0.032$ e 0.054 .

Em outras palavras, as transições para este caso é muito mais previsível do que para as topologias plano-parabólicas; assim, podemos dizer que as transições são mais controladas. Além disso, para a topologia plano-irregular temos apenas transições $L \rightarrow L+1$, enquanto que para as outras geometrias temos transições $L \rightarrow L+2$ ou $L \rightarrow L+1$.

\subsubsection{Dinâmica de Vórtices}

Passamos agora a discutir o movimento e dinâmica de vórtices em função do tempo e campo magnético aplicado. Em geral, a dinâmica é muito parecida com aquela para uma amostra plana descrita na Referência [35]. Nesta dissertação, nos concentraremos em aspectos que são substancialmente diferentes daqueles que ocorrem em amostras planas. Começaremos pela topologia plano-convexa para a vorticidade $L=2$ (ver Figuras 4.8). Inicialmente, os vórtices penetram ao longo de uma linha vertical; na medida em que o campo magnético aplicado vai aumentando, eles vão 
se aproximando, mas mantendo-se na direção vertical. Para um determinado valor de campo esses vórtices mudam de posição abruptamente se alinhando ao longo da diagonal da amostra. Isso não acontece para um filme plano; neste caso, logo que eles nucleiam dentro a amostra, eles imediatamente alinham-se ao longo da direção diagonal. Em outras palavras, antes de ocorrer a próxima nucleação, temos uma transição de fase estrutural de vórtices. Isto não é difícil de entender se considerarmos a topologia da amostra. Neste caso, uma rotação tem um custo energético uma vez que os vórtices tem de superar uma barreira de potencial intrínseca à topologia plano-convexa (ver Figura 4.1b). Como o campo magnético aplicado aumenta, esses dois vórtices vão se colapsar no centro, formando um vórtice gigante. Este novo estado se quebrará com a nucleação de mais dois novos vórtices.

Um cenário bem similar ocorre para vorticidade $L=4$, embora neste caso, a diferença entre o valor do campo de nucleação e o campo de transição de fase estrutural é muito pequena. Inicialmente os vórtices formam um quadrado com a diagonal perpendicular a um dos lados do quadrado supercondutor e permanecem nesta configuração para alguns valores de campo. Mas um pequeno aumento no campo aplicado força o sistema a acompanhar lateralmente os lados do supercondutor.

Na Tabela 4.1 resumimos todas as configurações de equilíbrio que obtivemos para todas as três topologias estudadas, para máximos valores de $\alpha, \beta$ e $\gamma$. Para interpretarmos corretamente os campos da tabela, seguiremos com a seguinte nomenclatura. Denotaremos por $L_{s} S$ uma configuração de multivórtices, formado por $L_{s}$ vórtices isolados. Um único vórtice gigante de vorticidade $L_{g}$ denotado por $1 G_{L g}$; o vínculo $L=L_{s}+L_{g}$ deverá ser obedecido. Por exemplo, o estado $4 S_{1} G_{2}$ indica quatro vórtices isolados formando um quadrado com um vórtice gigante duplamente quantizado no centro; $2 S$ é um estado para dois vórtices isolados alinhados com um dos 


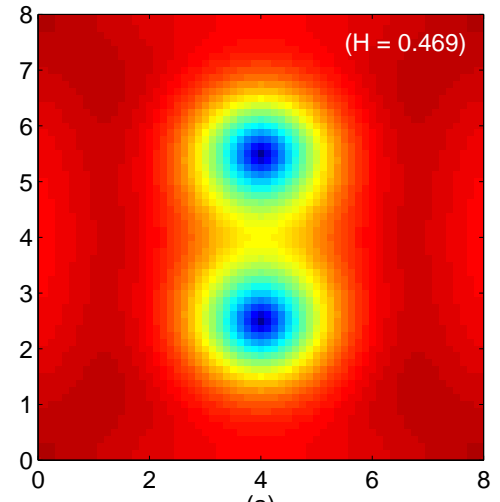

(a)

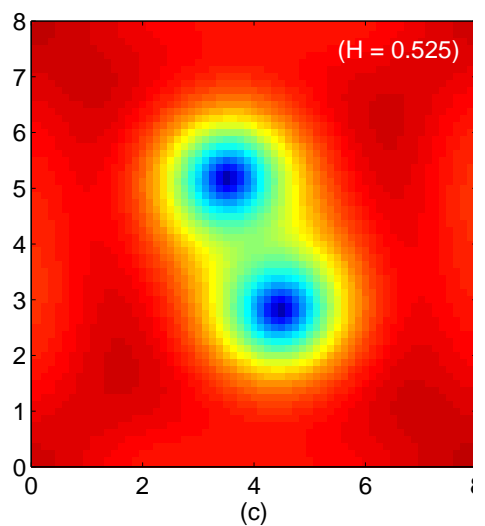

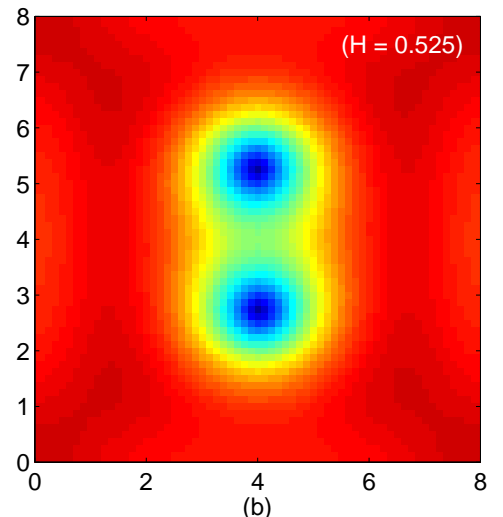

(b)

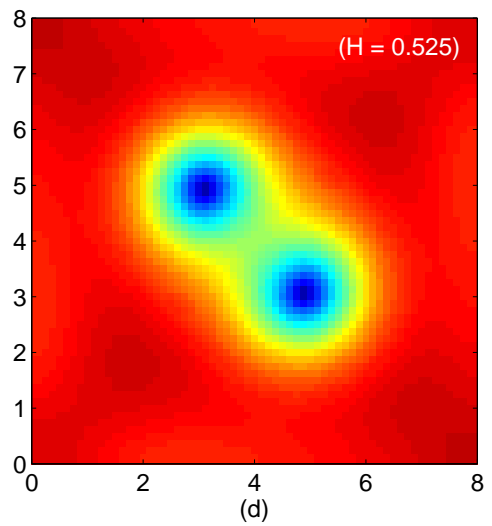

Figura 4.8: Dinâmica da movimentação de vórtices para vorticidade $L=2$ : topologia plano-convexa. 
lados do quadrado ou ao longo da diagonal; $3 S$ é um triângulo; $5 S$ é um quadrado com vórtices isolados e um no centro. Os estados $2 S, 3 S, 4 S$ e $5 S$ são levemente distorcidos para a topologia plano-irregular.

\begin{tabular}{llll}
\hline \hline$L$ & Plano-côncava & Plano-convexa & Plano-irregular \\
\hline 1 & - & - & $1 S$ \\
2 & $2 S \rightarrow 1 G_{2}$ & $2 S \rightarrow 1 G_{2}$ & $2 S$ \\
3 & - & - & $3 S$ \\
4 & $4 S$ & $4 S$ & $4 S$ \\
5 & - & - & $5 S$ \\
6 & $4 S 1 G_{2}$ & $4 S 1 G_{2}$ & $4 S 1 G_{2}$ \\
7 & - & - & $4 S 1 G_{3}$ \\
8 & $4 S 1 G_{4}$ & $4 S 1 G_{4}$ & $4 S 1 G_{4}$ \\
9 & - & - & $4 S 1 G_{5}$ \\
10 & $4 S 1 G_{6}$ & $4 S 1 G_{6}$ & $4 S 1 G_{6}$ \\
\hline \hline
\end{tabular}

Tabela 4.1: Configurações estáveis de vórtices para as três topologias. O significado dos campos da tabela é explicado no texto. A seta indica a evolução de um estado para o outro com o aumento do campo aplicado.

Um outro aspecto interessante nas configurações de vórtices é que para a topologia plano-irregular, podemos ter o estado de vórtices gigantes, mesmo para a rugosidade de $20 \%$. Isto porque há uma competição entre a rugosidade e a tendência na formação de um estado de vórtice gigante. A interação entre os vórtices é repulsiva, mas se a geometria da amostra apresenta dimensões muito reduzidas, ocorre a formação de vórtices gigantes. Como foi demonstrado na Referência [36] para um disco de superfície variável, a rugosidade da amostra evita a formação de vórtices gigantes. Porém, para este último caso, a simulação foi realizada para o caso de um disco de área ligeiramente maior que a área do quadrado usada nesta dissertação, ou seja, $8 \xi \times 8 \xi$. Para efeitos de comparação em pé de igualdade entre os dois casos, consideramos o aumento na área do quadrado para $9 \xi \times 9 \xi$, equivalente à área do disco usado na Referência [36]. Neste caso, observamos claramente que em situações onde ocorre vórtices gigantes para uma amostra plana, para uma rugosidade de $20 \%$ 
temos apenas estados de multivórtices. A Figura 4.9 confirma esta afirmação.

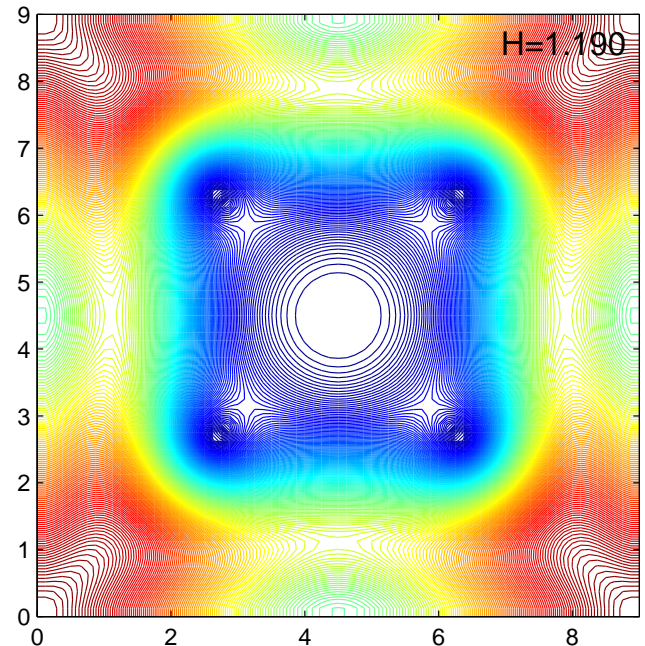

(a)

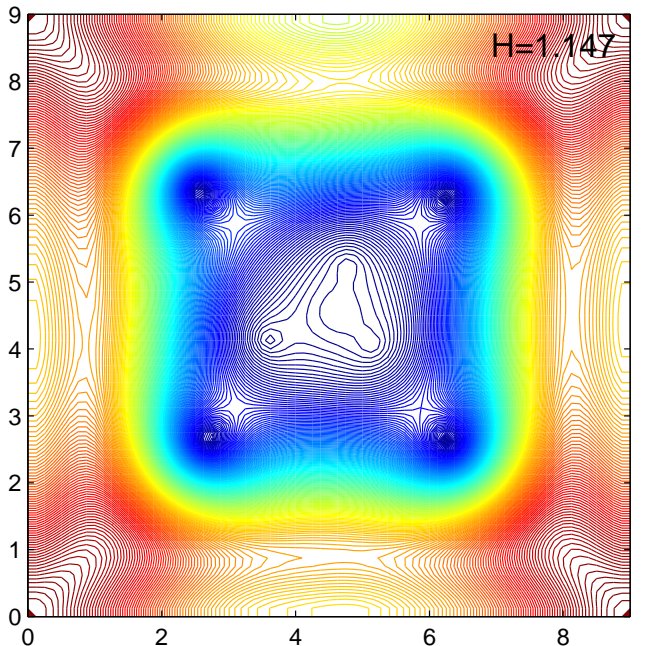

(b)

Figura 4.9: Densidade do parâmetro de ordem para $L=8$ : (a) superfície plana, (b) topologia plano-irregular com $20 \%$ de rugosidade. Para o quadrado plano, o campo de transição para esta vorticidade é $H=1.022$. Inicialmente os vórtices são distribuídos como num estado $8 S$ : os quatro vórtices mais externos, estão situados nos vértices de um quadrado, e os outros quatro vórtices, nos vértices de um quadrado interno, rotacionado de 90 graus com relação aos mais externos. Na medida em que o campo aplicado aumenta, os vórtices internos se movem para o centro se colapsando em um vórtice gigante como mostra o painel (a). Isto não ocorre na topologia plano-irregular como mostra o painel (b). A dinâmica da formação do estado correspondente ao painel (a) encontra-se na Figura 4.12.

Notamos também que, para a topologia plano-irregular de dimensões $8 \xi \times 8 \xi$, a acomodação de vórtices pode ser influenciada pelas diferentes formas irregulares que podem surgir para um mesmo valor de $\gamma$. Obtendo uma superfície tal que apresenta distância média entre os máximos da superfície irregular com valor igual ou maior do que $2 \xi$, acreditamos que pode ocorrer ancoramento. Essa escolha é feita por ser exatamente a dimensão do núcleo de um vórtice. Para isso, após várias tentativas, obtivemos uma superfície que apresenta uma distância média $d_{m}$ entre os máximos de 2.01 ; usando esta superfície pudemos notar que tal influência na acomodação de vórtices existe com relação a superfícies com valor menor de $d_{m}=1.73 \xi$. Os painéis 

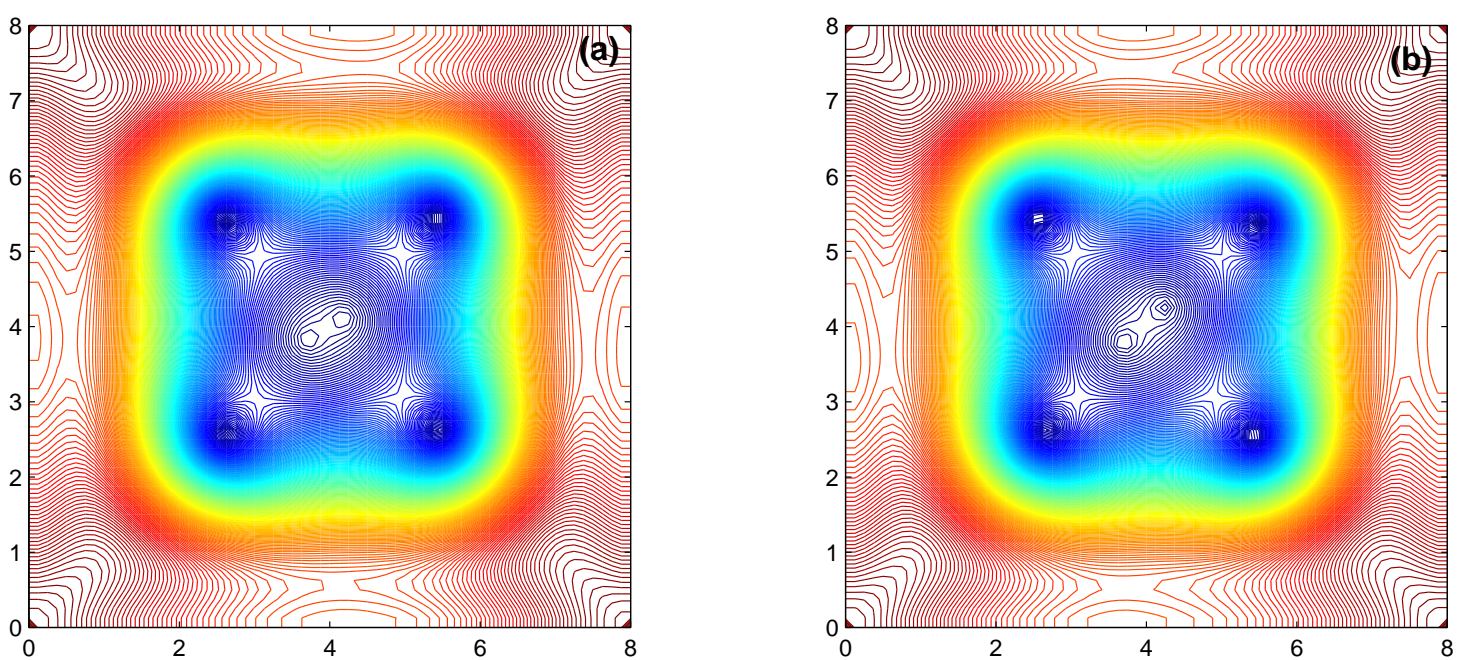

Figura 4.10: O painel (a) mostra um vórtice gigante no centro da amostra com $d_{m}=1.73 \xi$ e o painel (b) mostra dois vórtices isolados com $d_{m}=2.01 \xi$.

(a) e (b) da Figura 4.10, mostram estas diferanças. Podemos notar que em ambos os casos, os dois vórtices do centro aproximadamente se colapsam para formar um giante, embora rigosoamente falando eles não ocupam a mesma posição. No entanto, para o caso de maior valor de $d_{m}$ os vórtices tendem a ficar mais afastados. Por outro lado, ao fazermos a mesma análise para a topologia plano-irregular de dimensões $9 \xi \times 9 \xi$, com superfícies de diferentes valores de $d_{m}$, notamos apenas diferença na configuração dos vórtices do centro, mas em ambas as situações não observamos a formação de vórtices gigantes (ver Figura 4.11).

Muito recentemente, Nishio e colaboradores [20] conduziram um experimento usando microscopia de varredura (scanning Hall microcopy) num filme muito pequeno de $\mathrm{Pb}\left(4 \mu \mathrm{m}^{2}\right)$. A máxima vorticidade que eles puderam gerar foi de $L=5$. Mesmo para o estado $L=2$ eles não puderam observar um vórtice gigante conforme é previsto pela teoria de Ginzburg-Landau dependente do tempo para amostras planas. Embora eles não atribuam a dificuldade de observar o estado de vórtices gigantes devido à irregularidade da superfície do filme, nossos resultados sugerem fortemente que a rugosidade na superfície do filme é relevante e pode impedir a formação desse 

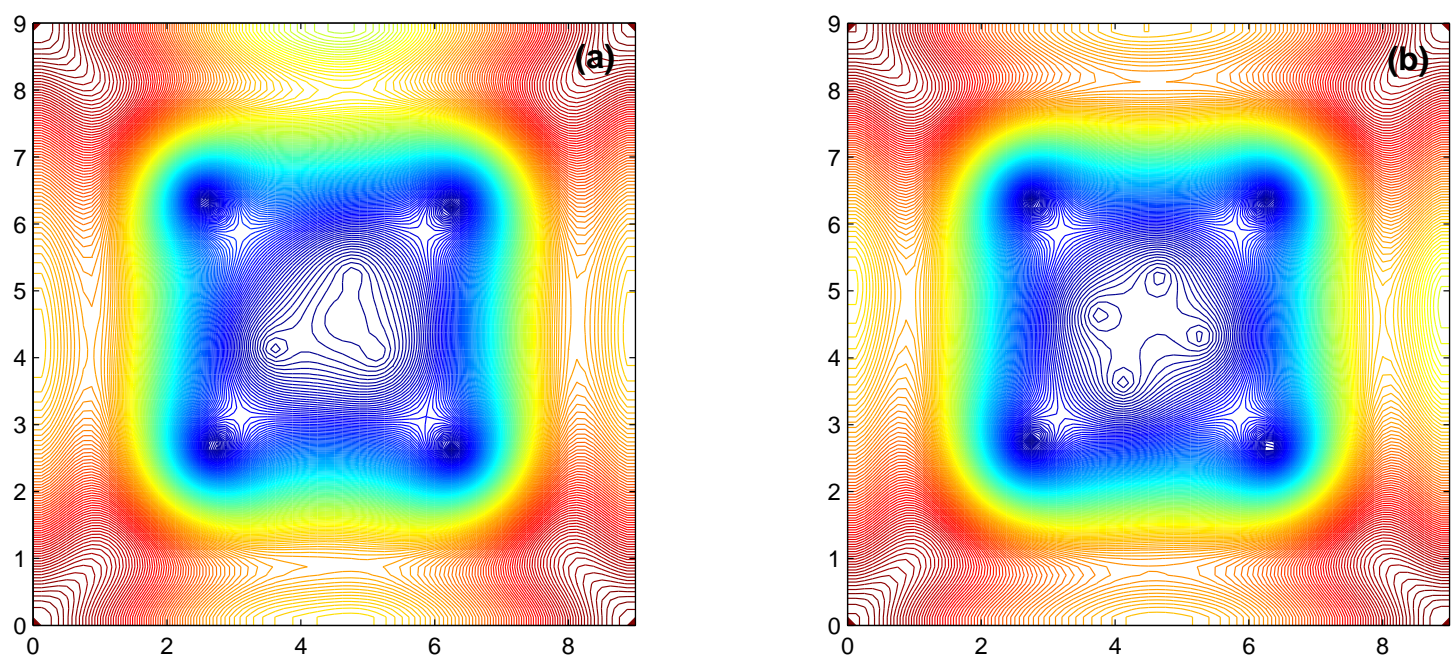

Figura 4.11: O painel (a) mostra o estado $L=8$ para uma superfície irregular com $d_{m}=1.40 \xi$ e o painel (b) a configuração do mesmo estado para uma superfície irregular com $d_{m}=2.10 \xi$.

tipo de estado.

Para finalizar, elucidaremos a dinâmica da evolução do estado $8 S$ ao estado $4 S_{1} G 4$ mostrado na Figura 4.9 a, na medida que aumentamos o campo magnético aplicado. Inicialmente temos dois estados $4 S$ com os quadrados rotacionados de $90^{0}$ um com relação ao outro. Conforme aumentamos o campo aplicado, os quatro vórtices do quadrado central mais e mais se aproximam um do outro, até colapsarem no centro. Algo bastante semelhante ocorre em um disco de raio $5 \xi$ (ver Figura 4.13). 


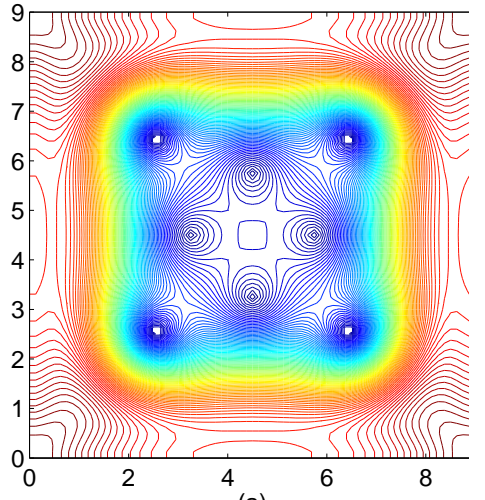

(a)

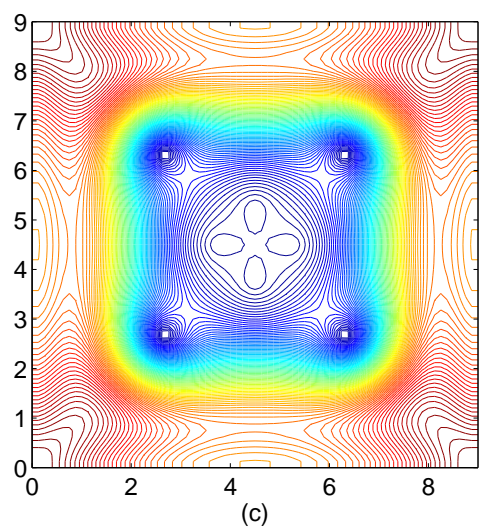

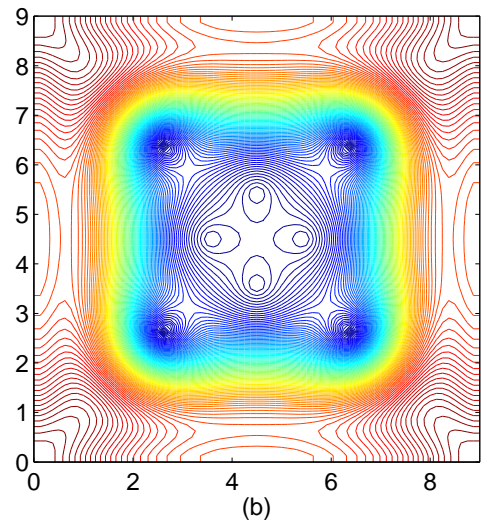

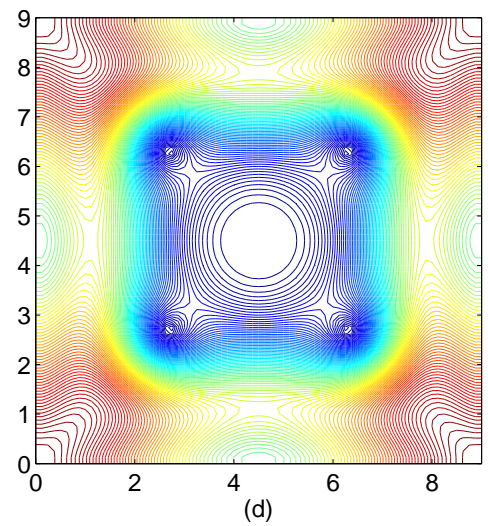

Figura 4.12: Topologia do parâmetro de ordem $\psi$ para a vorticidade $L=8$ : (a) $H / H_{c 2}=1.022$ (b) $H / H_{c 2}=1.079$, and (c) $H / H_{c 2}=1.126$; (d) $H / H_{c 2}=1.190$. 

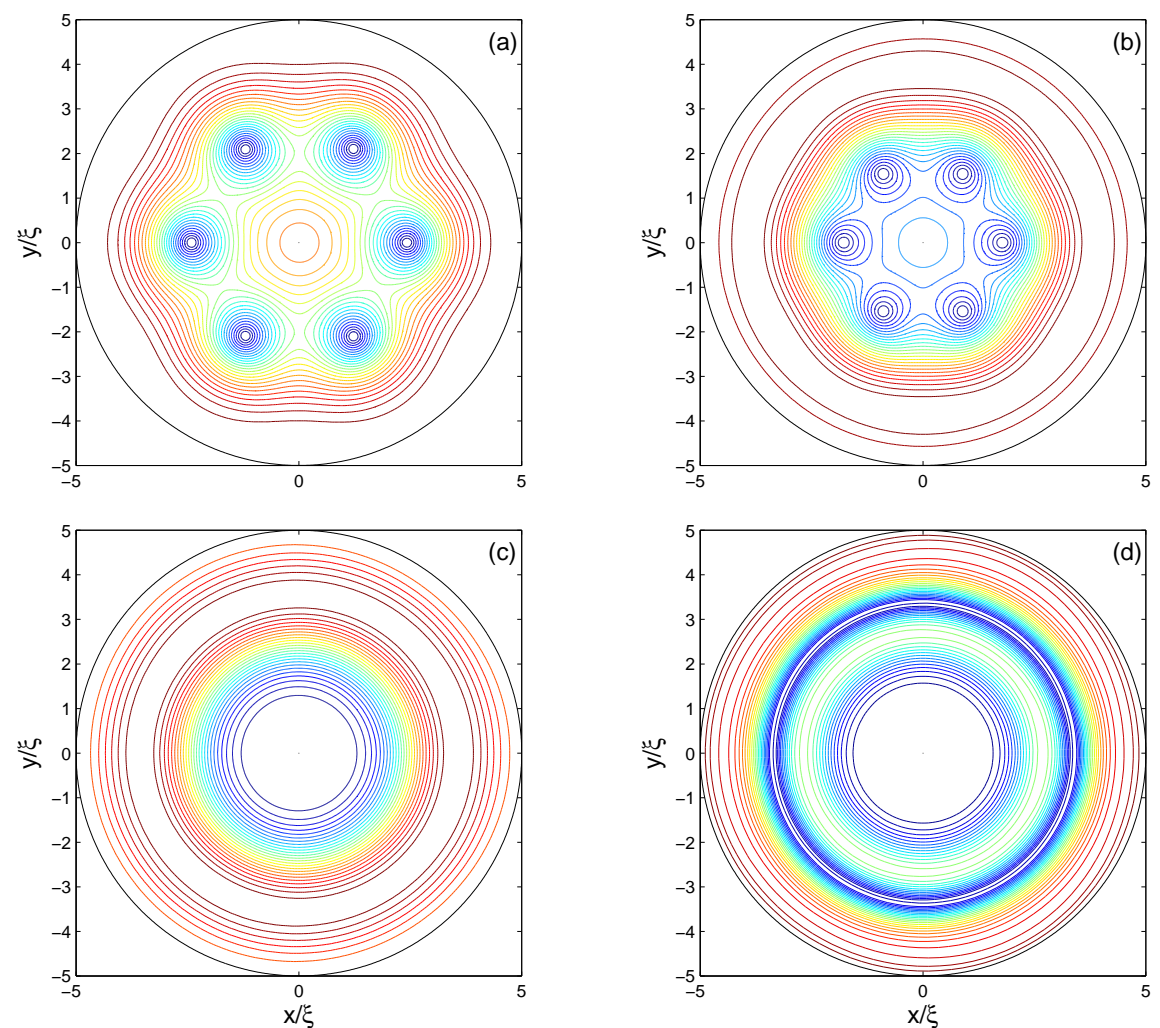

Figura 4.13: Topologia do parâmetro de ordem $\psi$ para a vorticidade $L=6$ : (a) $H / H_{c 2}=0.720$ (b) $H / H_{c 2}=0.950$, and (c) $H / H_{c 2}=1.064 ;$ (d) streamlines da densidade de corrente para $H / H_{c 2}=1.064$. 


\section{Capítulo 5}

\section{Conclusões}

Nesta dissertação, inicalmente fizemos uma revisão da literatura para o estudo introdutório da supercondutividade, no sentido se criar uma base teórica, de tal modo que possibilitasse aplicar os conceitos mais básicos a problemas mais complexos como simulações computacionais que permitem fazer previsões do comportamento de um supercondutor, tanto em nível macroscópico como mesoscópico. Começando pela proposta fenomenológia dos irmãos London, verificamos que este modelo é capaz de explicar o efeito Meissner e também a penetração superficial do campo magnético aplicado em materiais supercondutores. Em seguida, foi revisada a teoria de Ginzburg-Landau que, através de suas equações básicas, descrevem muito bem o comportamento de um supercondutor, ou seja, além do efeito Meissner e a penetração superficial do campo magnético, contempla os diferentes tipos de supercondutores e a dimensão dos vórtices.

Vimos também o método para resolver estas equações numericamente para supercondutores em estado de não-equilíbrio, que é conhecido como método das variáveis de ligação. Como já foi mencionado, com o uso de uma grade retangular de dimensões $N_{x} \times N_{y}$ células unitárias, por meio de uma escolha apropriada dos pontos da malha, pudemos discretizar as equações de Ginzburg-Landau dependentes do tempo de tal maneira a manter a invariância de calibre. 
Após este desenvolvimento inicial, foi feita em seguida uma revisão teórica para aplicar o método das variáveis de ligação a um problema específico, que foi para o estudo de filmes finos supercondutores. Vencida esta etapa, fizemos algumas adaptações ao código computacional que já havia sido desenvolvido para filmes planos o qual calcularia todos as quantidades físicas relevantes.

Ficou claro que a topologia do filme pode ter forte influência no comportamento do supercondutor quando imerso num campo magnético aplicado.

Ficou evidente que para amostras com topologia plano-côncava que apresentam concavidade mais acentuada a janela de supercondutividade é maior, ocorrendo o contrário para as amostras que apresentam convexidade maior. Observamos também que para ambas as topologias mencionadas acima, os saltos na magnetização são bem mais pronunciados do que para a toplogia plano-irregular. As transições para as topologias plano-parabolóides são mais alargadas, e ocorrem em menor número com relação às transições para a topologia plano-irregular. Pudemos notar também que as transições para esta última geometria ocorrem com maior regularidade com relação às demais; são todas do tipo $L \rightarrow L+1$, ao passo que para as outras topologias são híbridas (dos dois tipos $L \rightarrow L+1$, e $L \rightarrow L+2$ ). Notamos ainda que a largura das transições para a topologia plano-irregular são aproximadamente constantes.

Embora não tenhamos mostrado os laços de histerese completos, percebemos que nenhuma das topologias estudadas foi capaz de ancorar vórtices quando decrescemos o valor de campo aplicado passando por zero e invertendo sua polaridade. Desta forma, não pudemos observar colisões de vórtices com anti-vórtices ativados magneticamente.

Em relação ao aspecto computacional podemos concluir que trata-se de uma ferramenta muito poderosa. Além disso, uma vez escrito o código computacional 
aplicado a um problema específico, podemos extendê-lo para outras formas geométricas. Podemos também inserir defeitos distribuídos na amostra de forma regular ou irregular através da função $g(x, y)$.

Com isso, a perspectiva deste trabalho, seria analisar outras topologias com a intenção de encontrar amostras que apresente melhores performances supercondutoras. Por exemplo: transições mais estáveis, maior janela supercondutora, maior campo crítico superior, obtenção de centros de ancoramento de vórtices de tal forma que possamos ativar colisões vórtice-antivórtices e finalmente analisar com mais ênfase o efeito de confinamento, pois demostrou ser um efeito que provoca mudanças substanciais no comportamento supercondutor. 


\section{Referências Bibliográficas}

[1] Heike Kamerling Onnes. Communications from the Physical Laboratory at University of Leiden, 122B,124C, 1911.

[2] F. London and H. London. The electromagnetic equations of the supraconductor. Proceedings of the Royal Society of London. Series A, Mathematical and Physical Sciences, 149(866):71, 1935.

[3] Vitaly L. Ginzburg and L. Landau. Zh. Eksp. Teor. Fiz, 20:1064, 1950.

[4] J. Bardeen, L. N. Cooper, and J. R. Schrieffer. Theory of superconductivity. Physical Review, 108(5):1175, 1957.

[5] Leon N. Cooper. Bound electron pairs in a degenerate fermi gas. Physical Review, 104(4):1189, 1956.

[6] J. G. Bednorz and K. A. Muller. Zeitschrift für Physik B, 64:189, 1986.

[7] W. Meissner and R. Ochsenfeld. Ein neuer effekt bei eintritt der supraleitfahigkeit. Naturwinssenschaft, 21:787, 1933.

[8] Alexei A. Abrikosov. Magnetic properties of superconductors of the second group. Soviet Physics-Journal of Experimental and Theoretical Physics, 5:1174, 1957. 
[9] H. Träuble and U. Essmann. Direct demonstration of flux line movements in superconductors with current moving through them. Physica Status Solidi, 25:395, 1968.

[10] L. P. Gorkov. Microscopic derivation of the Ginzburg-Landau equations in the of superconductivity. Soviet Physics JETP-USSR, 9:1364, 1959.

[11] M. Tinkhan. Introduction to Superconductivity. McGraw-Hill Book Co, second edition, 2004.

[12] J. B. Ketterson. and S. N. Song. Superconductivity. Cambridge, University Press, 1999.

[13] A. L. Fetter and P. C. Hohenberg. Superconductivity, volume 2. Marcel Dekker, 1969.

[14] T. P. Orlando and K. A. Delin. Foundations of Applied Superconductivity. Addison-Wesley, 1990.

[15] George Arfken. Mathematical Methods for Physicists. Academic Press, 1970.

[16] Eugene Butkov. Física Matemática. Addison-Wesley, 1968.

[17] E. H. Brant. Thin superconductors and SQUIDs in perpendicular magnetic field. Physical Review B, 72:024529, 2005.

[18] B. J. Baelus and F. M. Peeters. Dependence of the vortex configuration on geometry of mesoscopic flat samples. Physical Review B, 65:104515, 2002.

[19] A. Kanda, B. J. Baelus, F. M. Peeters, K. Kadowaki, and Y. Ootuka. Experimental evidence for giant vortex states in a mesoscopic superconducting disk. Physical Review Letters, 93:257002, 2004. 
[20] T. Nishio, Q. Chen, W Gillijns, K. Keyser, K. Vervaeke, and V. V. Moshchalkov. Scanning Hall probe microscopy of vortex patterns in a superconducting microsquare. Physical Review B, 77:012502, 2008.

[21] E. Sardella, P. N. L-Filho, C. C. de S. Silva, L. R. E. Cabral, and W. Ortiz. Vortex-antivortex annihilation dynamics in a square mesoscopic superconducting cylinder. Physical Review B, 80:012506, 2008.

[22] G. M. Eliashberg. Soviet Physics JETP-USSR, 55:2443, 1969.

[23] L. P. Gorkov and G. M. Eliashberg. Generalization of Ginzburg-Landau equations for non-stationary problems in case of alloys with paramagnetic impurities. Soviet Physics JETP-USSR, 27:328, 1968.

[24] A. Schmid. A time dependent Ginzburg-Landau equation and its application to problem of resistivity in mixed state. Physik der Kondensiterten Materie, $5: 302,1966$.

[25] G. C. Buscaglia, C. Bolech, and A. López. Connectivity, volume m62. Springer, 2000.

[26] W. D. Gropp, H. G. Kaper, G. K. Leaf, D. M. Levine, M. Palumbo, and V. M. Vinokur. Numerical simulation of vortex dynamics in type-II superconductors. Journal of Computational Physics, 123:254, 1996.

[27] D. O. Gunter, H. G. Kaper, and G. K. Leaf. Implicit integration of the timedependent Ginzburg-Landau equations of superconductivity. SIAM-Journal on Scientific Computing, 23:1943, 2002.

[28] T. Winiecki and C. S. Adams. A fast semi-implicit finite-difference method for the TDGL equations. Journal of Computational Physics, 179:127, 2002. 
[29] J. W. Thomas. Numerical Partial Differential Equations. Texts in Applied Mathematics. Springer.

[30] S. J. Chapman, Q. Du, E. Lansing, and M. D. Gunzburger. A model for variable thickness superconducting thin films. Z Angew Math Phys, 47:410, 1996.

[31] S. J. Chapman and D. R. Heron. The motion of superconducting vortices in thin films of varyng thickness. SIAM-Society for Industrial and Applied Mathematics, 58:1808, 1998.

[32] Q. Du, M. D. Gunzburger, and J. S. Peterson. Computational simulation of type-II superconductivity including phenomena. Physical Review B, 51:16194, 1995 .

[33] E. Coskun. Computational simulation of flux trapping and vortex pinning in type-II superconductors. Applied Mathematics and Computation, 106:31, 1999.

[34] G. R. Berdiyorov, B. J. Baelus, M. V. Milosevic, and F. M. Peeters. Stability and transition between vortex configurations in square mesoscopic samples with antidots. Physical Review B, 68:174521, 2003.

[35] E. Sardella, P. N. L-Filho, A. L. Malvezzi, and W. Ortiz. Temperaturedependent vortex motion in a square mesoscopic superconducting cilynder: Ginzgurg-Landau calculations. Physical Review B, 74:014512, 2006.

[36] E. Sardella and E. H. Brandt. Vortices in a mesoscopic superconducting disk of variable thickness. Supercond. Sci. Technol., 23:025015, 2010. 


\section{Artigo Publicado Durante o Mestrado}

- M. C. V. Pascolati, E. Sardella, P. N. Lisboa-Filho, Physica C 470, 206 (2010). 